\title{
Non-Calcium Inorganic Materials Fabrication by Surface-Immobilized Organic Molecular Template
}

\author{
Peng Yang1, Wantai Yang ${ }^{1}$ Xu Zhang 2 and Jinchun Chen ${ }^{2}$ \\ ${ }^{1}$ College of Materials Science and Engineering, \\ Beijing University of Chemical Technology, Beijing, 100029, \\ ${ }^{2}$ College of Life Science and Technology, \\ Beijing University of Chemical Technology, Beijing, 100029,
}

P. R. China

\section{Introduction}

It has been extensively reported that organic molecules, i.e. biological molecules (CrookesGoodson et al, 2008) and polymers (Xu et al, 2007), could function as structural templating and/or selective adsorption at certain crystal faces, which may control and mediate the final crystal morphology. However, most of literatures are concentrated on the mediation ability of soluble free organic molecules in solutions. For practical applications, for example, tissue engineering, biomaterials fabrication and organic/inorganic hybrid film, a more straightforward way is to investigate such work on surfaces, that means, explore the mediation ability of surface-immobilized organic molecular template.

Until now, most of attentions are paid on the calcium-based inorganic material fabrication by such surface-immobilized organic molecular template (Tugulu et al, 2006; Kumar et al, 2010). However, besides calcium mineral, natural system also provides good biomineralization models for other kinds of useful inorganic materials such as silicate, barite, copper, iron and gold. The researches on the fabrication of these non-calcium materials by surface-immobilized organic molecular template have been not extensively investigated (Coffman et al, 2004; Kim et al, 2004 \& 2005; Morin et al, 2007). Therefore, our aim is to extend the content of the biomineralization mediated by surface-immobilized organic molecular template from classical calcium to a wide range of inorganic and metal materials through rationally engineering surface molecular template from small functional groups to macromolecules. We for the first time initiated this program in 2005 (Zhang et al, 2005) for the synthesis of silver nanoparticles mediated by surface-immobilized peptides, and after that, a series of researches in this field were reported to exploit the mediation ability of surface-immobilized organic molecule at different molecular scale, including small functional groups for the synthesis of titanium, zinc and silicon oxide films (Yang et al, 2007 and 2008; Gan et al, 2010), oligomer-grade peptides for the synthesis of gold, silver, platinum nanoparticles and superstructures (Zhang et al, 2006 and 2008; Wang et al, 2007; Li et al, 2007) and macromolecular brush for the fabrication of zinc oxide quantum dot and organic/inorganic hybrid nanocomposite film (Zou et al, 2009). 
In this chapter, we will systematically summarize the data obtained from our group. Engineered surface organic molecular template from our group could be categorized into three levels, depends on the size of surface immobilized molecule on substrates (Figure 1): level (1). small functional groups-mediated inorganic oxide deposition, growth and assembly; level (2). biomolecules such proteins, enzymes and peptides could be immobilized on small functional group-functionalized surface and these surfaceimmobilized biomolecules (i.e. inorganic-binding peptide) are further used as organic template to mediate noble metal deposition and growth; level (3). macromolecular (polymer) brush-mediated formation of semiconductor quantum dots in polymer matrix to fabricate organic/inorganic hybrid nanocomposites.

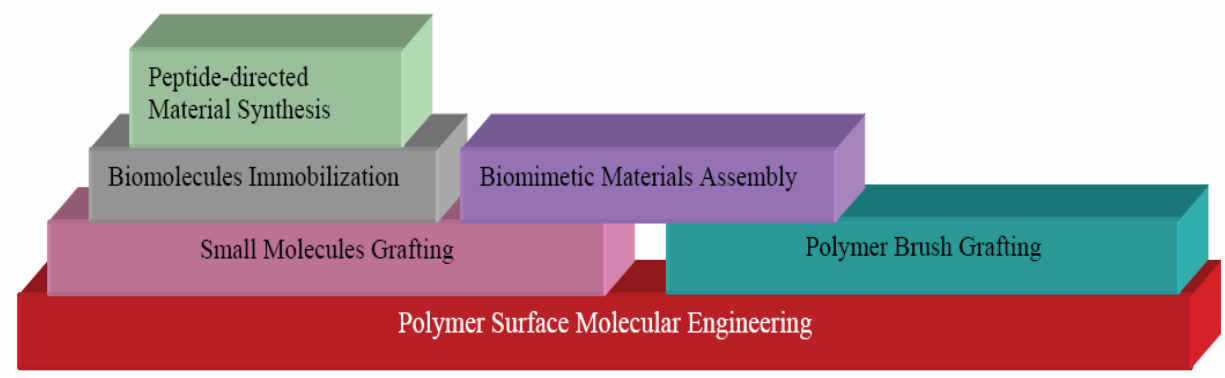

Fig. 1. Inorganic material fabrication mediated by surface-immobilized organic molecular template from our group. These templates could be categorized into three types: small molecules, peptide and polymer brush.

\section{Results and discussion}

\subsection{Level 1: Small molecule-mediated inorganic oxide film formation}

Firstly, surface-grafted small functional group is introduced. This is the smallest functional organic template for directing inorganic material deposition and growth on surfaces. Nevertheless, their mediation ability is not compromised by their tiny size and often very useful in advanced material and devices fabrication process. In 2003-2010, we continuously reported three novel confined photochemical reaction systems on polymer surface (Yang et al, 2003-2008; Gan et al, 2009 and 2010), which are eligible to convert directly C-H on polymer surface to well-defined reactive sulfate, hydroxyl and carboxyl monolayers. These organic functional groups are further used as molecular template to direct the nucleation and micro/nano-scale assembly of inorganic and metal oxides including silicon oxide $\left(\mathrm{SiO}_{\mathrm{x}}\right)$, titatnium dioxide $\left(\mathrm{TiO}_{2}\right)$ and zinc oxide $(\mathrm{ZnO})$. The resulting $\mathrm{TiO}_{2}$ and $\mathrm{ZnO}$ deposition could form isolated array (positive pattern) or continuous micronetwork (negative pattern, supported macroporous film). The latter is especially promising for preparation of highefficient solar cells because nanoparticles, photosensizers or complex salts could be conveniently incorporated into the holes of macroporous film from negative pattern. Moreover, this macroporous negative film has enough mechanical strength that supports its free transfer from original deposited support to any other substrates by simple adhesion technique. The resulting $\mathrm{SiO}_{x}$ deposition not only could form order array but also could form a transparent, ultraflat and continuous film on polymer substrate with widely tunable thickness and strong bonding strength. 


\subsubsection{Methods and chemistry to introduce functional small molecular monolayer on polymer surface}

In 2003, we for the first time found that when a very thin persulfate salt aqueous solution layer $(\mu \mathrm{m})$ was sandwiched between two polymer films and strong UV light irradiated the assembly from the side transparent to UV light, a fast surface hydrophilic modification method for most of commercial polymeric materials was developed (Figure 2) (Yang et al, 2003). For example, irradiating for $90 \mathrm{~s}$ and using $30 \mathrm{wt} \%$ ammonium persulfate, the static surface water contact angles of polymeric substrates decreased from $100^{\circ}$ to $44^{\circ}$ for low-density polyethylene (LDPE), from $107^{\circ}$ to $34^{\circ}$ for high-density polyethylene (HDPE), and from $73^{\circ}$ to $15^{\circ}$ for poly(ethylene terephthalate) (PET). The increases in surface hydrophilicity came from the formation of a sulfate salt group $\left(\mathrm{SO}_{4}-\mathrm{NH}_{4}{ }^{+}\right)$-ionized surface, which was characterized by $\mathrm{X}$-ray Photoelectron Spectroscopy (XPS) and Attenuated Total Reflection Fourier Transform Infrared spectroscopy (ATR-FTIR). The surface topography of the modified polymer substrates were observed by Scanning Electron Microscope (SEM) and Atomic Force Microscope (AFM), and no visible etching effects to original surface were found.

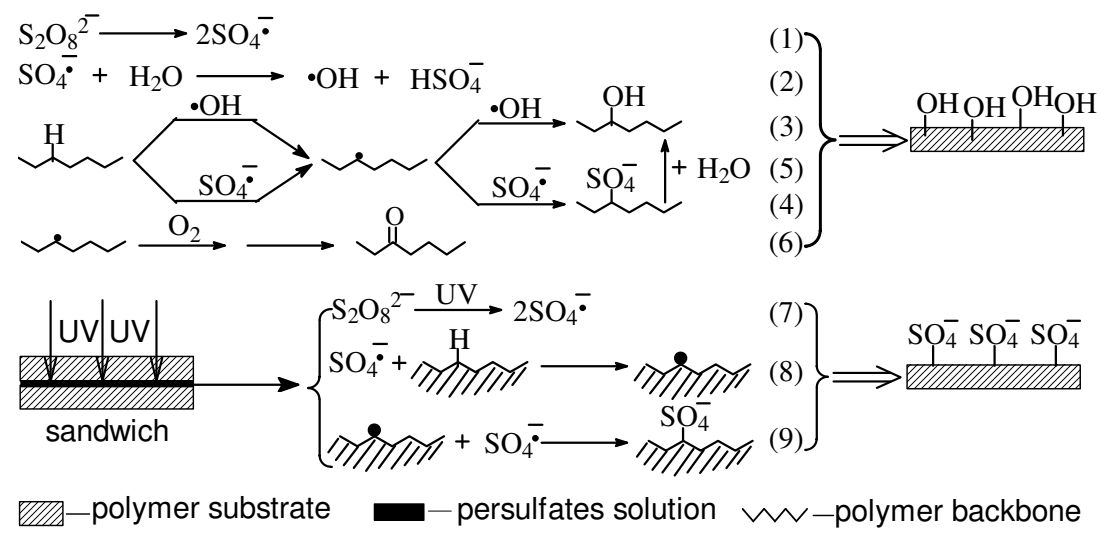

Fig. 2. The possible reaction model for surface oxidation by persulfates: the conventional and the "sandwich" methods. Reproduced from (Yang et al, 2003). Copyright from Elsevier. Reproduced with permission.

A possible reaction model named by 'confined photo-catalytic oxidation' (CPO) was put forward to interpret the above results. Conventional photooxidation route of persulfate is that water $\left(\mathrm{H}_{2} \mathrm{O}\right)$ molecules are firstly oxidized by persulfates to form hydroxyl radicals, which undertake further oxidization to organic substances. However, in such sandwiching setup, due to the role of $\mathrm{CPO}$, a new reaction route is achieved where persulfates dissociate directly to form sulfate anion radicals under UV irradiation, the resulting sulfate radicals quickly oxidize polymer surface by abstracting hydrogen atoms from polymer surface, and then the formed surface radicals couple with other sulfate anion radicals to fabricate an array of sulfate anion groups covalently attached on the outmost surface. This strategy provides an effective surface hydrophilic and functionalization method suitable for most of commercial polymeric materials with the advantages of fast, mild (to environment and substrate), facile and low cost technological process. Based on CPO, we further develop a very simple method to create hydrophilic/hydrophobic hybrid polymer surface, through 
"CPO" under the regional control by a photomask (Figure 3). The resulting wettability or surface molecular template patterns have been used to fabricate polyaniline (PANI) (Yang et al, 2006), $\mathrm{TiO}_{2}$ and $\mathrm{ZnO}$ micropatterns.
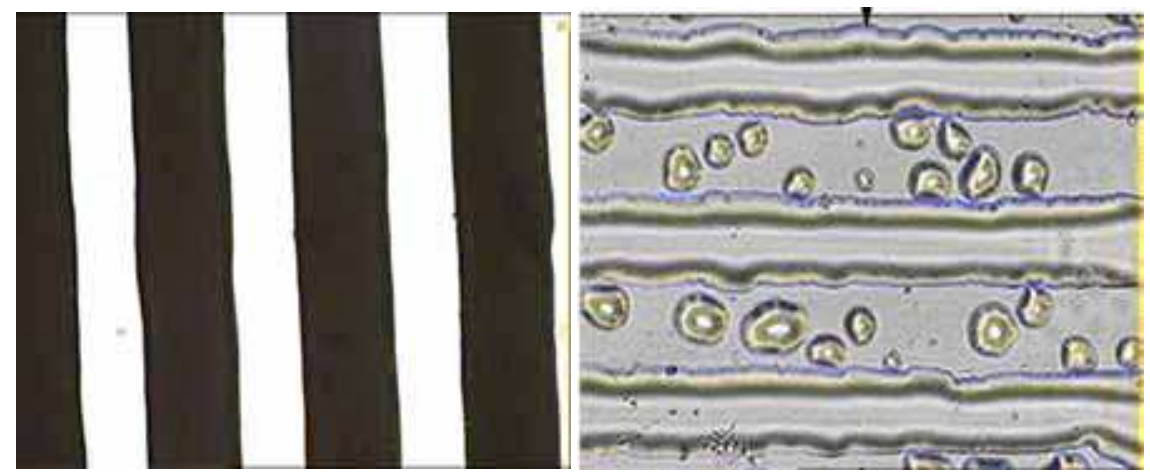

Fig. 3. The wettability pattern obtained by CPO method. The left image is a photomask used in CPO reaction to control the irradiation/non-irradiation areas: the white bars are UV transparent regions which will result in the corresponding UV-exposed regions (i.e. CPO reaction occurred) on polymer surface, producing a hydrophilic region, as shown in parallel water condensation lines in right image; in contrast, the black regions in the photomask are metallic parts which will shield UV light and thereby result in non-irradiated parts on underlying polymer substrate, producing original hydrophobic surface, as shown in right image (the spaces between hydrophilic lines). Reproduced from (Yang et al, 2006). Copyright from Wiley-VCH Verlag $\mathrm{GmbH} \& \mathrm{Co}$. KGaA. Reproduced with permission.

Moreover, the formed sulfate groups on surfaces could be readily hydrolyzed to form hydroxyl monolayer, which has been demonstrated by XPS, contact angle (CA) measurement (Figure 4) and ATR-FTIR (Figure 5). As shown in Figure 4, when sulfated surface was soaked in water, the surface contact angle increased gradually and reached a plateau after about 10 hours. It has been known that polymer surfaces hydrophilized by oxidizing treatments should not decay when store in water or high-energy media. The conflict between present results and this theory revealed that surface sulfate groups actually converted to hydroxyl groups during this soaking process because of the difference in surface free energy between the sulfate anion and hydroxyl groups. The conversion of sulfate to hydroxyl group-implanted surfaces resulted in the continuous increase of the CA on the treated surface and became constant when the hydrolysis reached equilibrium. This judgment was further strongly supported by ATR-FTIR (Figure 5). We used a classical reaction to tell the existence of hydroxyl groups on the surface, that is, allowed the sulfated surface after soaking in water to be incubated in trifluoroacetic anhydride (TFAA) solution under certain conditions. If there are a plenty of hydroxyl groups on the surface, such incubation would result in an effective reaction between surface hydroxyl group and TFAA in the solution, producing characteristic ester and C-F signals in ATR-FTIR spectra. The results were as we expected. The spectrum of the sample after soaking (c) displayed an ester carbonyl bond at $1789 \mathrm{~cm}^{-1}$ and a C-F bond at $1222 \mathrm{~cm}^{-1}$, both of which could not be found in the spectra of the samples before soaking $(b, d)$ and the sample for the untreated LDPE (a). XPS (data not shown here) was also consistent with ATR-FTIR results, and both of them 


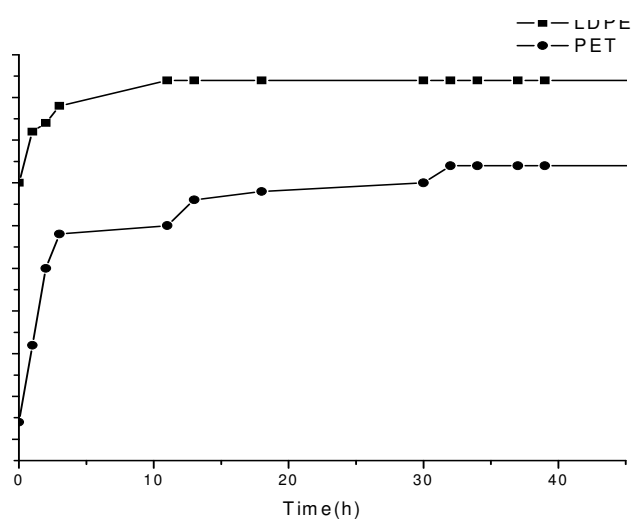

Fig. 4. Surface hydrophilicity changes upon soaking modified surface in water. Reproduced from (Yang et al, 2007). Copyright from American Chemical Society. Reproduced with permission.

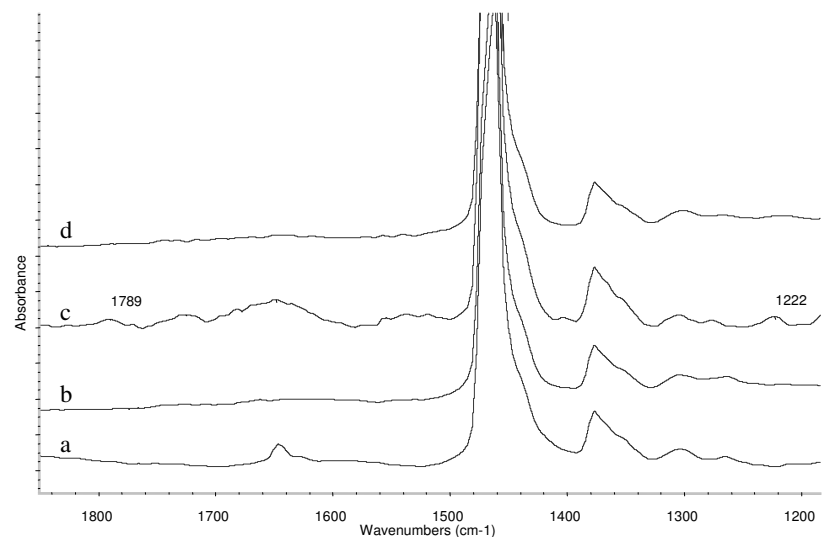

Fig. 5. ATR-FTIR spectra: (a) LDPE: unirradiated; (b) LDPE: irradiated; (c) the derivation of modified LDPE: irradiated, soaked into water for $14 \mathrm{~h}$, and then treated with TFAA at $30^{\circ} \mathrm{C}$, dried under reduced pressure at $20^{\circ} \mathrm{C}$ for $24 \mathrm{~h}$; (d) the derivation of modified LDPE:

irradiated, and then treated with TFAA at $30^{\circ} \mathrm{C}$, dried under reduced pressure at $20^{\circ} \mathrm{C}$ for 24h. (Irradiation conditions: LDPE films; irradiation time is $90 \mathrm{~s}$; the conc. of $\left(\mathrm{NH}_{4}\right)_{2} \mathrm{~S}_{2} \mathrm{O}_{8}$ is $30 \%$, UV-intensity is $6500 \mu \mathrm{w} / \mathrm{cm}^{2}$ ). Reproduced from (Yang et al, 2007). Copyright from American Chemical Society. Reproduced with permission.

$$
\mathrm{SO}_{4}^{-}
$$

Fig. 6. Hydrolysis reaction scheme of grafted sulfate groups on polymer surface. 
plus CA measurements indicated strongly that the polar sulfate groups on the CPO-treated surface converted to hydroxyl groups after slow hydrolysis in water (Figure 6).

After successfully introducing anion sulfate and neutral hydroxyl groups on surfaces, we also developed two novel approaches to introduce amine groups on surfaces, because these amine groups could potentially provide positive charge and reactivity towards proteins and silanes. The chemistry and methods used to fabricate such aminated surface are based on newly-developed UV-induced Surface Aminolysis Reaction (USAR) and silanization on
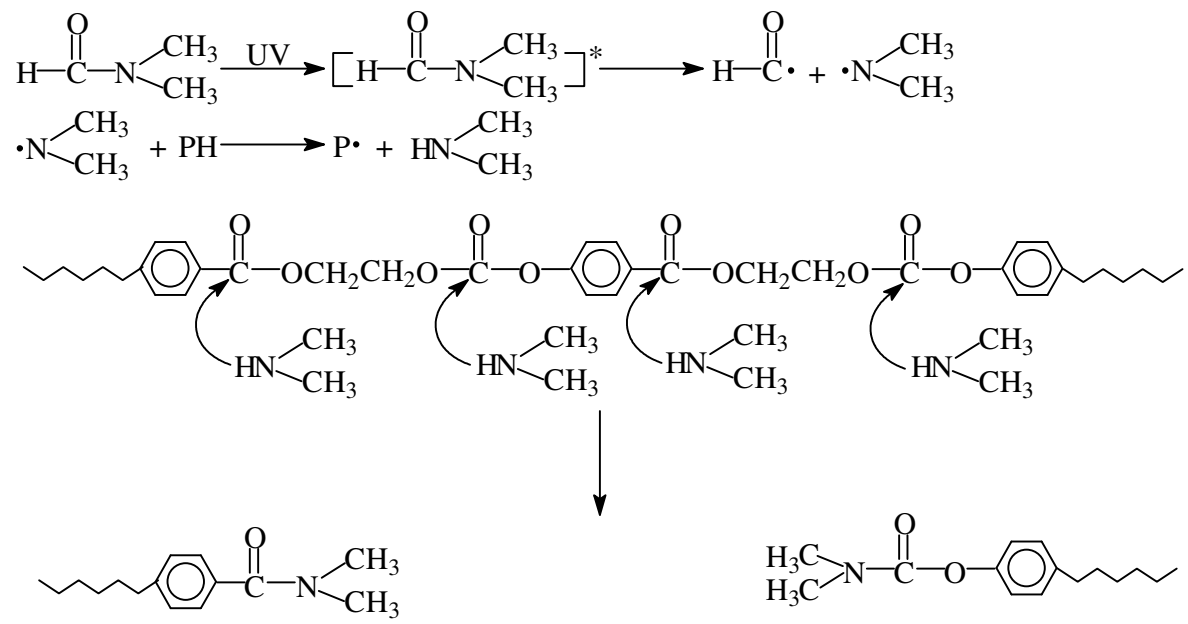

$\mathrm{HOCH}_{2} \mathrm{CH}_{2} \mathrm{OH}$<smiles>CN(C)C(=O)c1ccc(C(=O)N(C)C)cc1</smiles>

$\mathrm{HOCH}_{2} \mathrm{CH}_{2} \mathrm{OH}$

Fig. 7. A plausible reaction mechanism of USAR to introduce positive amine on PET surface. Reproduced from (Yang et al, 2005). Copyright from Wiley-VCH Verlag GmbH \& Co. KGaA. Reproduced with permission.

hydroxyl-modified surface by CPO. In the former reaction, a thin film of $\mathrm{N}, \mathrm{N}$ dimethylformamide (DMF) liquid is sandwiched between two polyester films, and then transferred to UV field for irradiation. It has been known that dimethylamine and formaldehyde would be released very slowly when DMF is irradiated by visible light. However, in such confined reaction setup, this reaction is obviously accelerated largely. Systemic investigations reveal that after UV irradiation for short time (2-16min), DMF undergoes photodissociation to give out amine molecules (dimethylamine), which could insitu attack ester bond along PET chains to incorporate tertiary-amine functional groups at PET chain end (Figure 7). With additional photomask, the region to be aminated could be designed and controlled with micron-meter precision, as shown in Figure $8 \mathrm{~b}$ by selective staining from Sumikaron red dye. After positive amines incorporation, we further demonstrated that peptides, enzymes and proteins could be immobilized on such surface with keeping their biological activity for catalysis and biomineralization. For example, as shown in Figure 8c, when the enzyme, horseradish peroxide (HRP), was immobilized on aminated surface (circular regions), the enzyme could effectively catalyze the reaction with the standard substrate, 3-amino-9-ethylcarbazole (AEC) to produce precipitations which 
was deposited on circular aminated regions (line-type particles). Besides this direct aminolysis on polyester surface, a more general approach is also developed by us to introduce primary amine groups on most of polymer surface. This is a derivation reaction on hydroxyl-modified surface by CPO, that is, when hydroxyl monolayer is formed on surfaces by $\mathrm{CPO}$, the further silanization reaction with any other silane reagent could give us a well-defined functionalized surface (Gan et al, 2009). We have used this kind of surface to immobilize antibody effectively.

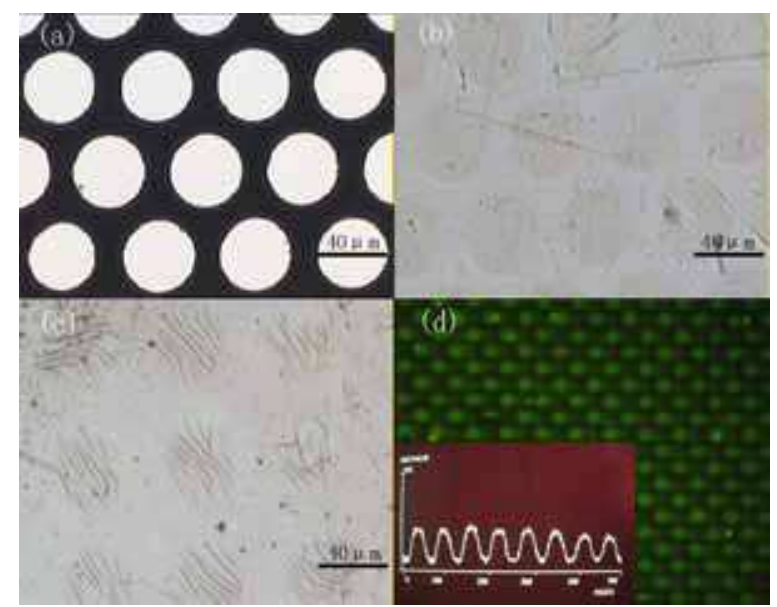

Fig. 8. (a). Micrograph of metallic(Cr) photomask. the diameter of circle hole is $40 \mu \mathrm{m}$. (b). Micrograph of Sumikaron red stained microprocessing surface. Irradiation time for USAR is $16 \mathrm{~min}$, and aminated film was stained by a water solution of Sumikaron red, followed by ultrasonic washing. (c). Micrograph of patterned aminated surface after electrostatic selfassembling HRP. (d). Fluorescent micrograph of patterned aminated surface after electrostatic self-assembling IgG. Irradiation time for USAR is $16 \mathrm{~min}$. The inset in Figure 8(d) was the line profile of fluorescence intensity of the FITC-IgG pattern in Figure 8(d). Reproduced from (Yang et al, 2005). Copyright from Wiley-VCH Verlag GmbH \& Co. $\mathrm{KGaA}$. Reproduced with permission.

\subsection{2 $\mathrm{TiO}_{2}$ film fabrication on sulfated and hydroxylated polymer surface by biomimetic nucleation and growth in aqueous solution. (Yang et al, 2007)}

For plastic electronics and optics, the fabrication of smooth, transparent and stable crackfree inorganic oxide films (and patterning) on flexible polymeric substrates with strong bonding strength and controllable thickness from nanometers to micrometers is a key but still remains a challenge. From this section, we will continuously introduce a series of novel approaches developed by us to fabricate high quality $\mathrm{TiO}_{2}, \mathrm{ZnO}$ and $\mathrm{SiO}_{x}$ films on polymer substrate by a mild biomimetic interface-directed nucleation and growth process.

The fabrication of $\mathrm{TiO}_{2}$ films on substrates especially flexible polymers is extremely important due to their great potential in photocatalysis, energy conversion, and electrooptical techniques. Such films are often fabricated by sol-gel method where gelation at high temperature is needed to ensure the quality of crystals. In contrast, low-temperature biomimetic nucleation and growth in aqueous solution by surface-immobilized functional 
groups could provide an alternative way to fabricate high quality $\mathrm{TiO}_{2}$ film on weak polymer substrate where high temperature annealing must be avoided.

For this aim, we firstly fabricated a wettability-patterned polypropylene surface by CPO. As shown in Figure 9, after CPO modification, the biaxially oriented polypropylene (BOPP) film surface was incubated in a mixture solution of $\left(\mathrm{NH}_{4}\right)_{2} \mathrm{TiF}_{6}$ and $\mathrm{H}_{3} \mathrm{BO}_{3}$ at certain low temperature, molar ratio and incubation time. Under deposition condition (c), an anatase $\mathrm{TiO}_{2}$ film could be selectively deposited on a hydrophilic region with keeping original hydrophobic regions being undisturbed (no deposition). Figure 10 presented an optical microscope image of this positive pattern. AFM analysis indicated that the thickness of such film under deposition condition (c) was $350 \mathrm{~nm}$. A 3D AFM profile image further showed that the obtained $\mathrm{TiO}_{2}$ film consisted of crystal fusions from dense column arrays. Such column-type crystal growth implied that the deposition process was dominated by a preferential $\mathrm{c}$ axis (normal line direction on the surface) oriented crystallization growth. (Masuda et al, 2003; Dutschke et al, 2003; Strohm et al, 2005). The resulting patterned $\mathrm{TiO}_{2}$ film possessed significant bonding strength with underling substrates and good line edge acuity. Actually, when standard 3M adhesive tape peeling experiment was applied on this positive pattern film, the film could sustain very well. Such selective deposition could be attributed to the preferential nucleation and growth on sulfate group implanted (i.e. irradiated hydrophilic) regions. This functional group-mediated inorganic oxide deposition has been observed and developed in selfassembled monolayer (SAM) on inorganic and metal substrates, and our approach provided an alternative way to do this on polymer substrate where SAM is difficultly constructed. Obviously, this biomimetic approach provided an effective solution toward the microfabrication on various inert polymer substrates.

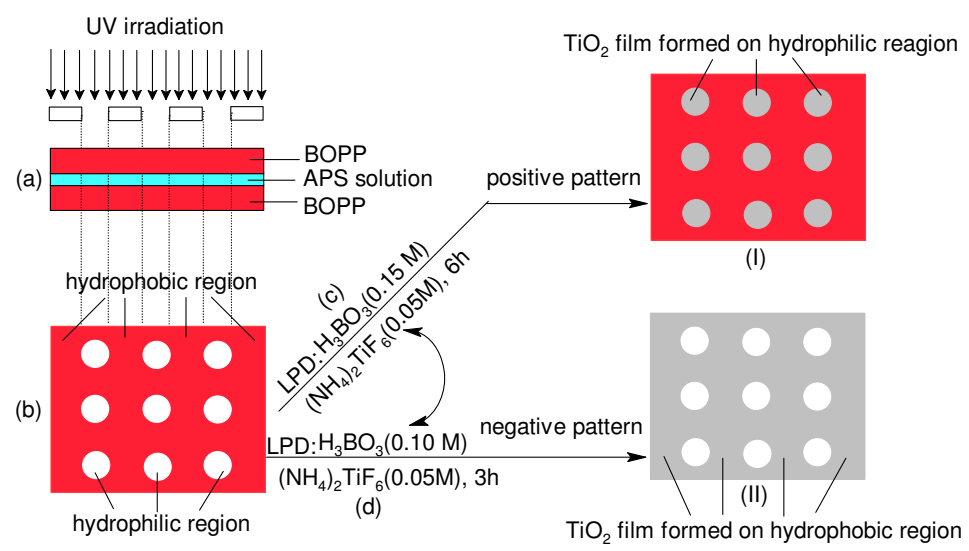

Fig. 9. The schematic process of $\mathrm{TiO}_{2}$ micropattern on BOPP film. (a). A thin layer of ammonium persulfate (APS) aqueous solution was sandwiched between two BOPP films, and selective UV irradiation was conducted by using a photomask; (b). CPO took place in the irradiated region, and the resulting wettability-patterned surface was utilized to fabricate positive (I) pattern under positive condition (c) and negative (II) pattern under negative condition (d) respectively. Reproduced from (Yang et al, 2007). Copyright from American Chemical Society. Reproduced with permission. 

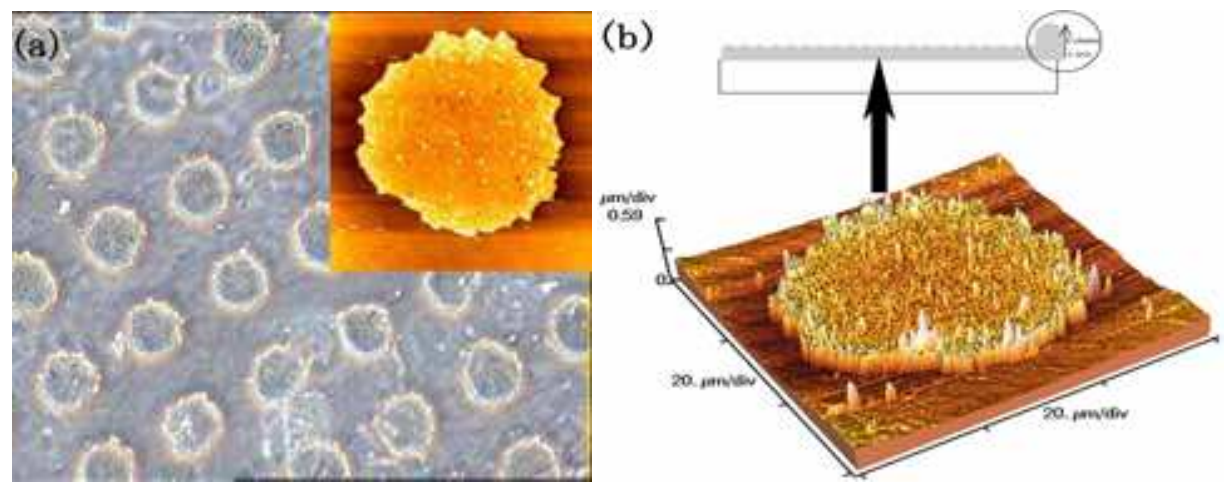

Fig. 10. Optical and AFM images of positive pattern on BOPP surface obtained by condition (c). (a). Optical image of positive pattern, the inert picture in (a) showed a detailed circle $\mathrm{TiO}_{2}$ deposition layer by AFM; (b). 3D profile AFM image of positive pattern (a), the upper picture in (b) showed the schematic column growth of $\mathrm{TiO}_{2}$ crystalline particle along c-axis, this column-pattern could be found clearly in the lower 3D profile AFM picture. Reproduced from (Yang et al, 2007). Copyright from American Chemical Society. Reproduced with permission.
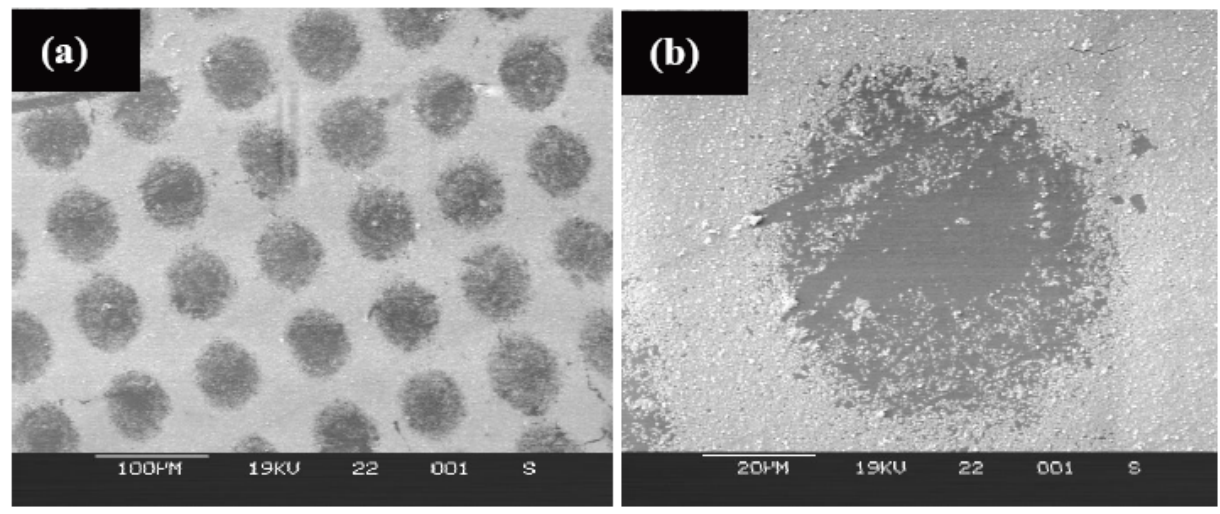

Fig. 11. SEM images of the negative $\mathrm{TiO}_{2}$ pattern on $\mathrm{BOPP}$ surface obtained by condition (d): (a). SEM image of negative pattern; (b). an enlarged image in circle region in (a). Reproduced from (Yang et al, 2007). Copyright from American Chemical Society. Reproduced with permission.

More surprisingly, we also found, for the first time, that under deposition condition (d), instead of deposition on hydrophilic regions, the deposition of $\mathrm{TiO}_{2}$ film would take a phase inversion process, that is, the deposition was only selectively retained on the hydrophobic region with keeping hydrophilic regions being undisturbed (no deposition) to form a negative pattern. Figure 11 presented a typical negative pattern obtained by such method. This is a very interesting and novel phenomenon found firstly by us, and further investigation on the mechanism showed that the hydrolysis reaction of active sulfate groups and the resulting affinity change towards $\mathrm{TiO}_{2}$ crystals during this process played a key role 
(Figure 12). Such negative patterns refute the conventional opinion that only hydrophilic regions favor the formation of $\mathrm{TiO}_{2}$ films and could be used to fabricate large areas $\left(\mathrm{mm}^{2}\right)$ of interconnected $\mathrm{TiO}_{2}$ micronetworks, which are obviously difficult to obtain by conventional metallic masks. Such negative pattern also could be freely transferred to any other substrate by adhesive tape peeling (Figure 13) and could be used as a flexible photomask for photochemical reactions (Figure 14).

To summary, the present method that surface functionality mediated $\mathrm{TiO}_{2}$ deposition is expected to provide new strategies in the fabrication of flexible positive $\mathrm{TiO}_{2}$ array and negative macro/mesoporous $\mathrm{TiO}_{2}$ interconnected films on flexible organic substrates, without the use of complex photolithography procedures. We further found that this method is also suitable for fabrication of other inorganic oxide films such as $\mathrm{ZnO}$ and $\mathrm{SiO}_{2}$, as described below. Further exploration on other kinds of inorganic oxide film fabrication by such method and incorporation of functional molecules into the positive film and the pores formed in negative film for functional device fabrication may be suggested as the next direction of this research.

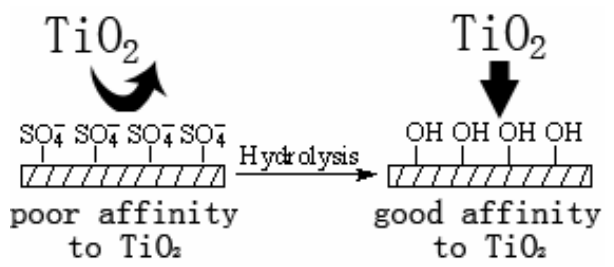

Fig. 12. The chemical change of functional groups on oxidized surface by hydrolysis reaction. It was found that sulfate groups had poor affinity to titanium layer $\left(\mathrm{TiO}_{2}\right)$, while hydroxyl groups had good affinity to $\mathrm{TiO}_{2}$. Reproduced from (Yang et al, 2007). Copyright from American Chemical Society. Reproduced with permission.

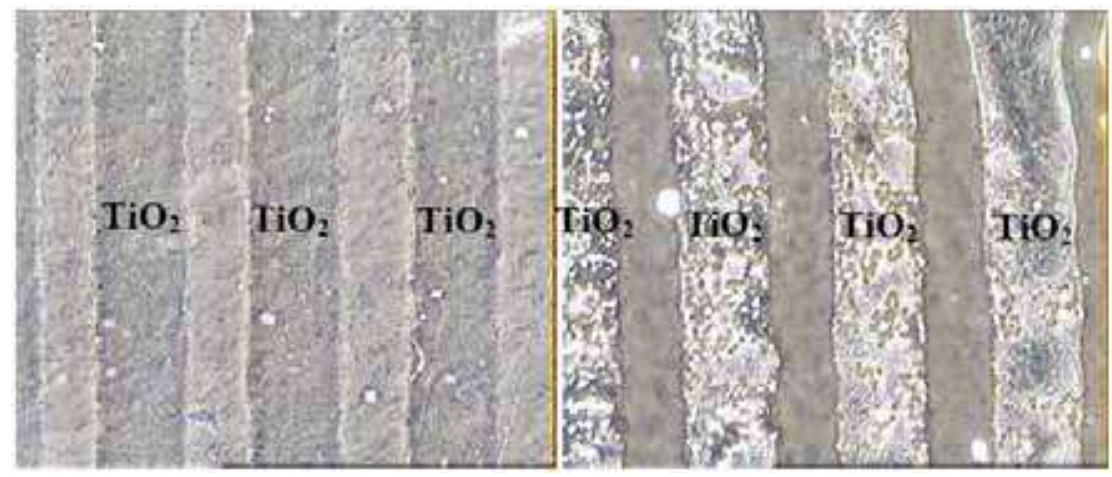

Fig. 13. Negative $\mathrm{TiO}_{2}$ pattern formed on BOPP substrate and subsequent peeling for transferring such pattern onto an adhesive tape. (a). optical images of the negative $\mathrm{TiO}_{2}$ micropattern formed on BOPP surface by condition (d) (using a photomask with parallel line pattern). (b). optical images of the negative $\mathrm{TiO}_{2}$ micropattern on the $3 \mathrm{M} \mathrm{Scotch}^{\circledR}$ adhesive tape after peeling the $\mathrm{TiO}_{2}$ pattern in (a) from BOPP substrate. Reproduced from (Yang et al, 2007). Copyright from American Chemical Society. Reproduced with permission. 


\subsubsection{ZnO film fabrication on sulfated, hydroxylated and caroxylated polymer surfaces} by biomimetic nucleation and growth in aqueous solution. (Yang et al, 2008)

Similar to patterned $\mathrm{TiO}_{2}$ deposition, patterned $\mathrm{ZnO}$ deposition on polymer substrates has also received great attentions because of its promising potential in photocatalysis, energy conversion, and electro-optical techniques. Our research indicated the functionality pattern formed by $\mathrm{CPO}$ method could be also used to mediate the growth of $\mathrm{ZnO}$ crystals on polymer substrates under mild conditions, giving positive and negative patterns on BOPP and PET substrates respectively (Figure 15). The fabrication was achieved by incubating functionalized polymer film in a mixture solution of $\mathrm{Zn}\left(\mathrm{NO}_{3}\right)_{2}$ and hexamethylenetetramine (HMT) at suitable temperature and concentration (Yang et al, 2008).
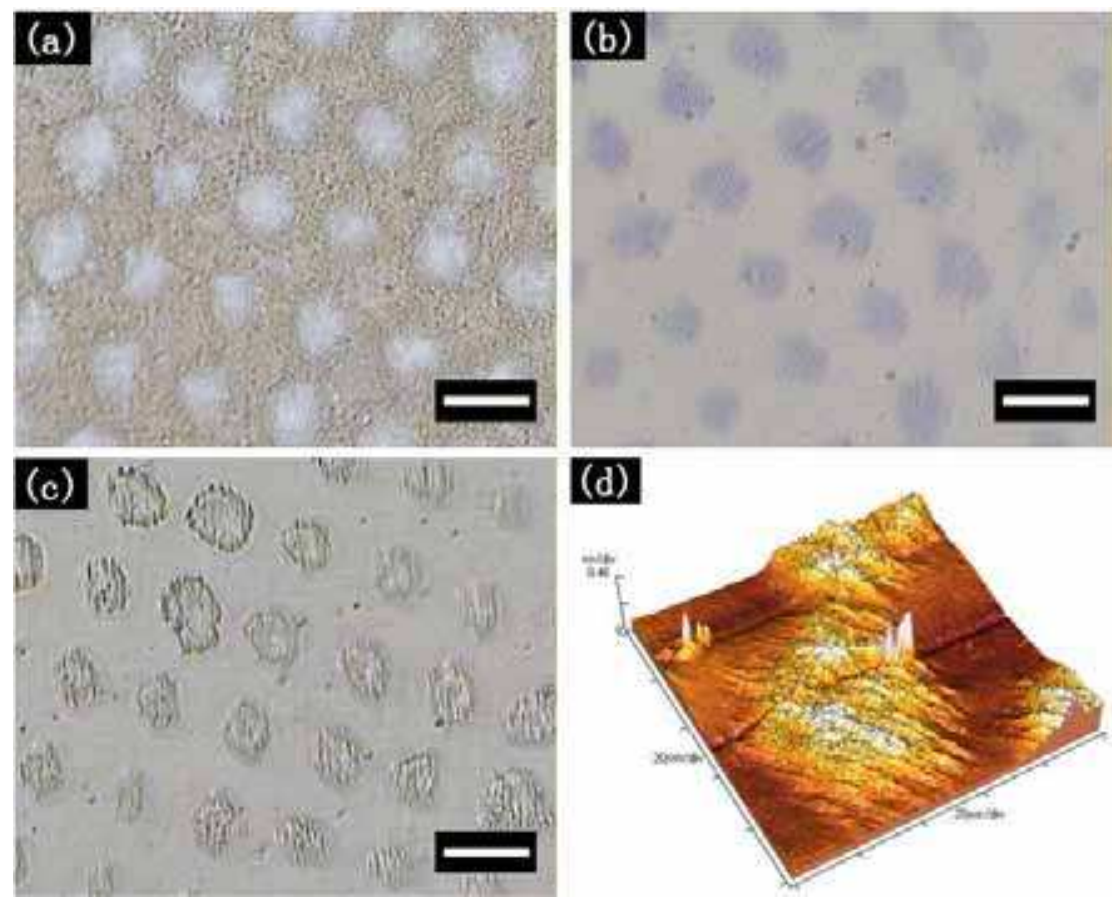

Fig. 14. Micropatterning of a BOPP surface with patterned photograft polymerization products of acrylic acid (AA) by using the negative $\mathrm{TiO}_{2}$ pattern on the BOPP film as a photomask. (a). Phase contrast microscope image of the negative $\mathrm{TiO}_{2}$ pattern on the $\mathrm{BOPP}$ film. (b). Optical microscope image of the patterned poly(AA) (PAA) grafts on the BOPP surface after staining with toluidine blue. (c). Phase contrast microscope image of the patterned PAA grafts on the BOPP surface. (d). 3D AFM image of a circle from the PAA grafted region on the BOPP surface. The scale bars in (a)-(c) are $80 \mu \mathrm{m}$. Reproduced from (Yang et al, 2007). Copyright from American Chemical Society. Reproduced with permission.

Similar to the column-type growth behavior observed in $\mathrm{TiO}_{2}$ deposition system, $\mathrm{ZnO}$ film also grew along c-axis from the surface. However, fusion among these columns is not observed in $\mathrm{ZnO}$ material, and separated $\mathrm{ZnO}$ rods are obtained in the resulting $\mathrm{ZnO}$ film. 
We found that the rods having the typical size around 500-750nm in diameter and $2.5 \mu \mathrm{m}$ in length, was selectively obtained on sulfated and hydroxylated regions of BOPP substrate, resulting in a positive $\mathrm{ZnO}$ pattern which was corresponding to the irradiated regions by UV (Figure 16 and 17). In contrast, for reactive polyesters such as PET, the ZnO rods selectively remained on the unmodified original regions, creating negative patterns which were corresponding to the unirradiated regions (Figure 18 and 19). X-ray Diffraction (XRD) pattern was further used to characterize the crystal morphology of $\mathrm{ZnO}$ rods obtained on sulfated and hydroxylated BOPP as well as PET surfaces (Figure 20). Interestingly, different dominating crystal direction was found on four kinds of surfaces. On BOPP-OH surface, preferential c-axis direction was found since (002) plane had the maximum intensity. When this substrate was changed to $\mathrm{BOPP}-\mathrm{SO}_{4}{ }^{-}$, this c-axis orientation was lowered and as a result, both of c-axis direction as (002) and nearly parallel directions to the substrate as (100), (101) co-dominated in the crystal morphology. When the substrate was further changed to PET from BOPP, dominated crystal direction was found around the direction parallel to the substrate since the planes as (100) and (101) had the higher signal intensity. The detailed reason for this substrate-dependent crystal morphology change is needed to be investigated in the future, and different crystallization orientation on original BOPP and PET films may become a possible reason.

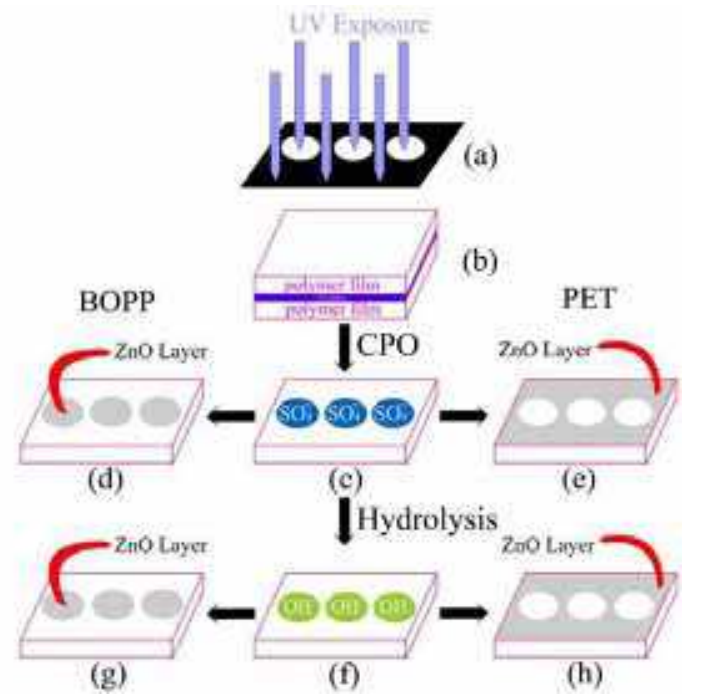

Fig. 15. The growth scheme of the $\mathrm{ZnO}$ layer on patterned functionalized polymer surfaces: (a) UV rays transported selectively through a photomask with circular holes; (b) a sandwich setup consisting of two polymer films with a persulfate ammonium solution in the middle, irradiated by UV light whose route was controlled by the photomask; (c) following the patterned irradiation, the sulfate groups could be selectively grafted onto the irradiated regions, which could be further transformed into hydroxyl groups by a hydrolysis reaction; (f) the $\mathrm{ZnO}$ layer selectively remained on the sulfated and hydroxylated regions of the BOPP film (d, g), whereas for PET, the growth of the ZnO layer selectively remained on the unirradiated regions (e, h). Reproduced from (Yang et al, 2008). Copyright from Wiley-VCH Verlag $\mathrm{GmbH} \& \mathrm{Co}$. KGaA. Reproduced with permission. 
Moreover, the photoluminescence property of a $\mathrm{ZnO}$ crystal was also investigated. Near UV and visible luminescence from $\mathrm{ZnO}$ crystals were detected by fluorescence spectroscopy (Figure 21). Strong UV and visible-light luminescence originated from band edge emission $(395 \mathrm{~nm})$ and the oxygen vacancy $(450-600 \mathrm{~nm})$ respectively (Masuda et al, 2006; Wu et al, 2001; Kang et al, 2003), showing a potential for polymer-based display devices. The mechanism for the above-mentioned pattern formation also involves in the mediation ability of surface functional monolayers. On BOPP substrates, functionalized sulfated and hydroxylated regions were found to favor the formation of a $\mathrm{ZnO}$ layer through possible electrostatic and hydrogen coordination between functional groups and $\mathrm{ZnO}$ crystals (Chaudhary et al, 2004; Zubkov et al, 2005; Saito et al, 2001). As mentioned above, the sulfate groups on the surface were not stable species which would undertake a slow hydrolysis to give hydroxyl groups. This process also released protons as byproduct $\left(\mathrm{H}^{+}\right)$(Yang et al, 2003; House et al, 1962; Wilmarth et al, 1962; Bamford et al, 1994). This newly formed $\mathrm{H}^{+}$
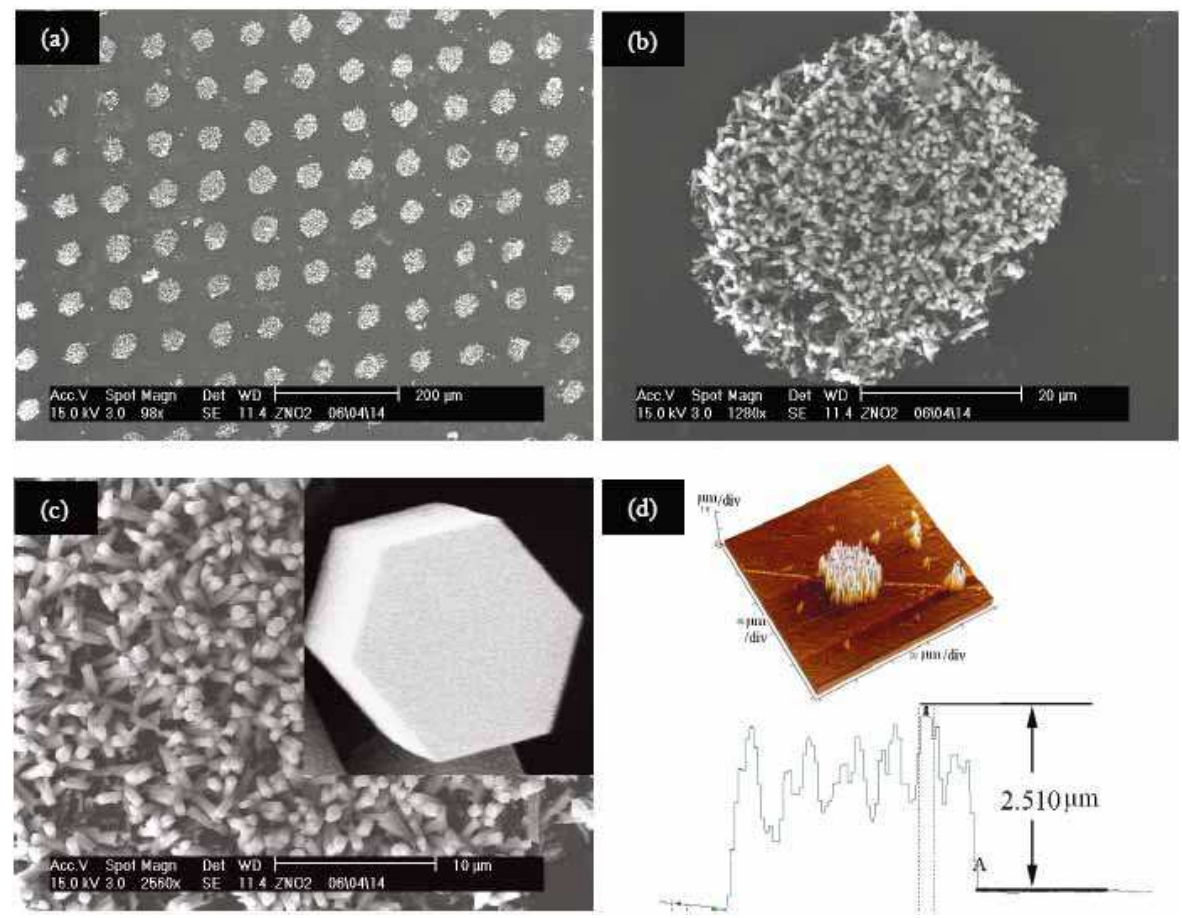

Fig. 16. (a-c) SEM and (d) AFM images of a patterned $\mathrm{ZnO}$ deposition on a sulfated BOPP surface via ultrasonic washing. (a) The resulting film showed a regular circle pattern where the $\mathrm{ZnO}$ layer could be found solely on the irradiated region. The magnifications $(\mathrm{b}, \mathrm{c})$ showed that the diameter of the deposited region and a single $\mathrm{ZnO}$ pillar was about $40 \mu \mathrm{m}$ and $680 \mathrm{~nm}$, respectively. The cross-sectional shape of the ZnO pillar was typically hexagonal (inset picture in (c)). (d) An AFM image of a single circular pattern indicated a thickness of the $\mathrm{ZnO}$ layer on the sulfated area of about $2.5 \mu \mathrm{m}$. Reproduced from (Yang et al, 2008). Copyright from Wiley-VCH Verlag GmbH \& Co. KGaA. Reproduced with permission. 
would decrease the extent of supersaturation of the solution as a result of neutralization with $\mathrm{OH}$ - formed by the hydrolysis of HMT. Accordingly, during the same time scale, the extent of deposition reaction for $\mathrm{ZnO}$ growth on the sulfated BOPP surface would be lower than that on the hydroxylated BOPP surface. This analysis was well demonstrated by the following experiment: under equivalent conditions $\left(0.1 \mathrm{M}, 90^{\circ} \mathrm{C}, 48 \mathrm{~h}\right)$, the density of the $\mathrm{ZnO}$ rods on a sulfated BOPP surface was $0.8 / \mu \mathrm{m}^{2}$, whereas this value increased to $1.3 / \mu \mathrm{m}^{2}$ on the hydroxylated surface.

For the negative patterns on PET substrates, Koumoto et al (Masuda et al, 2006) suggested the reason for this was because the $\mathrm{ZnO}$ crystal surface could become hydrophobic by the adsorption of certain hydrophobic organic molecules used in the solution, and then the hydrophobic $\mathrm{ZnO}$ crystallites naturally preferred to be deposited on the hydrophobic unirradiated PET surface. We analyzed this phenomenon from another point of view, that is, the effect of surface functional group plays an important role on this pattern formation.
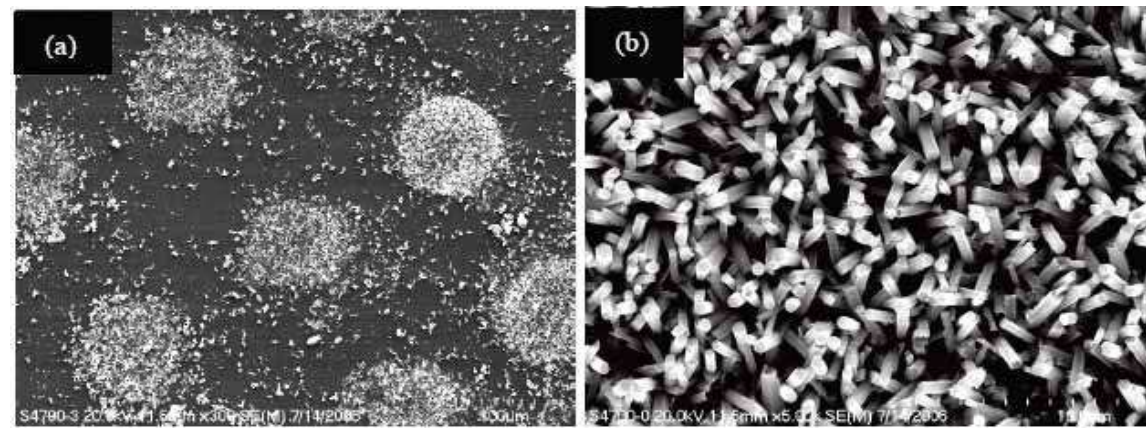

Fig. 17. (a) A SEM micrograph of a patterned ZnO deposition on a hydroxylated BOPP surface and (b) a magnification of one circular region. By ultrasonic washing, the ZnO pillar became selective to the hydroxylated BOPP region whereas a few particles dispersed into the unirradiated region. The average diameter of the $\mathrm{ZnO}$ rods was $500 \mathrm{~nm}$. Reproduced from (Yang et al, 2008). Copyright from Wiley-VCH Verlag GmbH \& Co. KGaA. Reproduced with permission.

Not like inert BOPP, PET is a relatively reactive substrate containing abundant ester bonds, which are capable to undergo hydrolysis reactions in aqueous environments (Karayannidis et al, 2007). Similar to our consideration, Pizem et al (Pizem et al, 2005) also pointed out that during the solution deposition process of a titanium film on polyimide substrates, reactive imide groups could be partially hydrolyzed and thereby influence the acidic oxide deposition solution. In our work, we found that the existence of polar sulfate and hydroxyl groups on irradiated PET regions would facilitate the alkaline hydrolysis to release more $\mathrm{COOH}$ groups in a weak base environment than unirradiated regions, because grafting of sulfate and hydroxyl groups onto the $a$ site of the glycol ester (COOCH2CH2OOC) along PET chains would effectively increase the rate constant of the alkaline hydrolysis (Schmeer et al, 1990; Bruice et al, 1961 and 1962), whereas this effect was not obvious on unirradiated regions without such polar groups. The existence of $\mathrm{COOH}$ groups on substrates would weaken the deposition of the $\mathrm{ZnO}$ layer (Hsu et al, 2005) because these $\mathrm{COOH}$ endgroups on polymer surface could firstly be deprotonated and negatively charged, and then preferentially bonded with HMT molecules to create electrostatic and/or steric screening for 

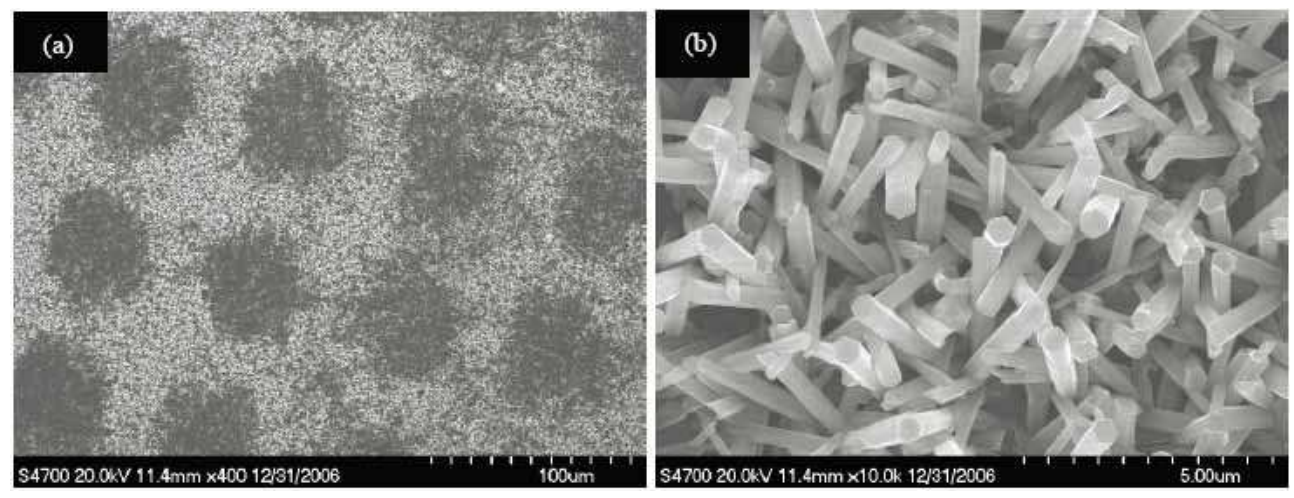

Fig. 18. SEM micrographs of ZnO deposition on patterned sulfated PET surface and the magnification displaying the detailed deposition on sulfated (the dark regions in (a)) and unirradiated regions ((b) and the bright regions in (a)). Very little deposition was found on the sulfated regions represented by a circular pattern. Rather, the deposition was mainly found to form a continuous network on the unirradiated regions. The average diameter of the $\mathrm{ZnO}$ rods was about $750 \mathrm{~nm}$. Reproduced from (Yang et al, 2008). Copyright from Wiley-VCH Verlag GmbH \& Co. KGaA. Reproduced with permission.
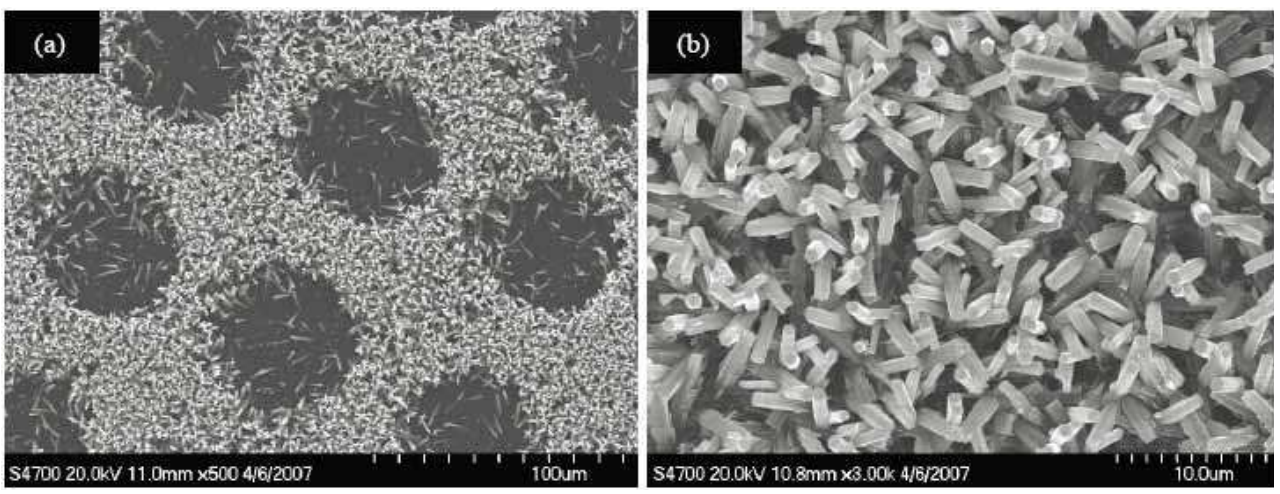

Fig. 19. SEM micrographs of the ZnO deposition on a patterned hydroxylated PET surface and the magnification displaying the detailed deposition on hydroxylated (the dark regions in (a)) and unirradiated regions ((b) and the bright regions in (a)). The average diameter of $\mathrm{ZnO}$ rods was about $750 \mathrm{~nm}$. Reproduced from (Yang et al, 2008). Copyright from Wiley$\mathrm{VCH}$ Verlag $\mathrm{GmbH} \& \mathrm{Co}$. KGaA. Reproduced with permission. 

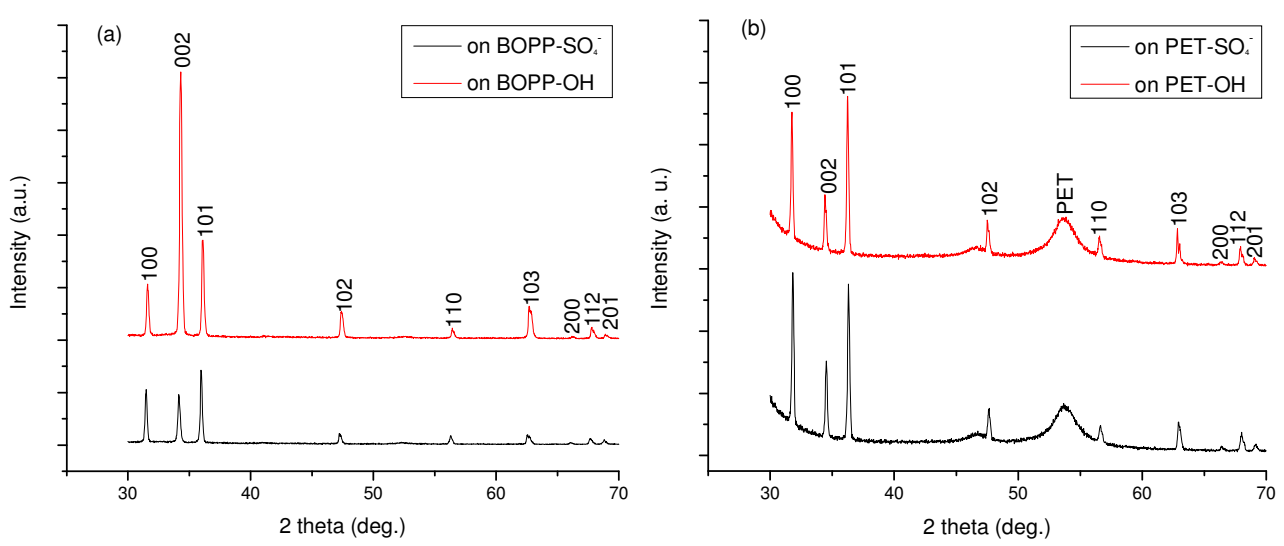

Fig. 20. XRD patterns of $\mathrm{ZnO}$ rods deposited on substrates of (a) sulfated BOPP (lower curve in (a), BOPP-SO $4^{-}$), hydroxylated BOPP (upper curve in (a), BOPP-OH), and (b) sulfated PET (lower curve in (b), PET-SO $4^{-}$) and hydroxylated PET (upper curve in (b), PET-OH). Reproduced from (Yang et al, 2008). Copyright from Wiley-VCH Verlag GmbH \& Co. $\mathrm{KGaA}$. Reproduced with permission.
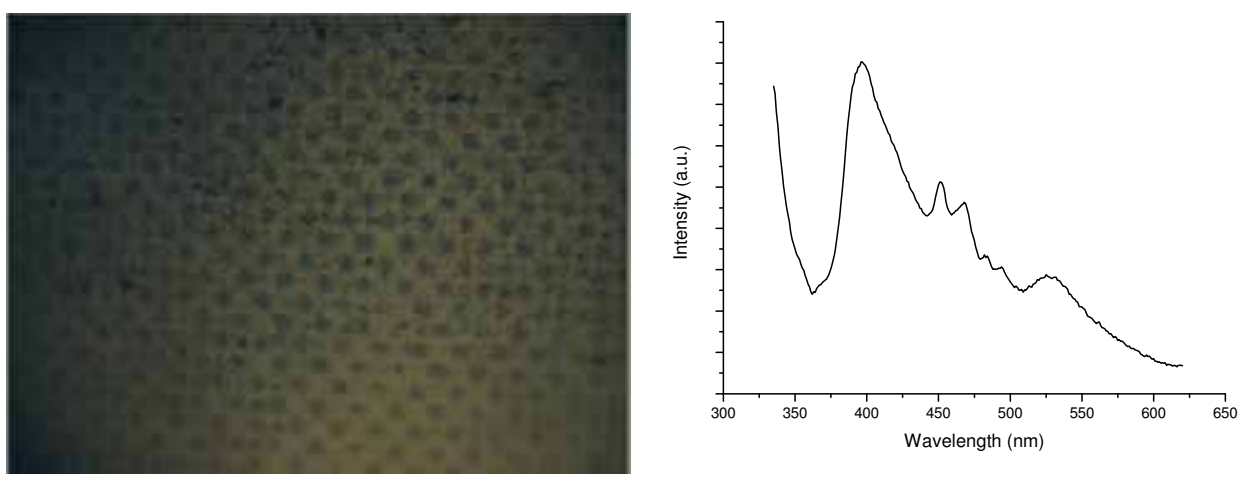

Fig. 21. A photoluminescence image of a patterned $\mathrm{ZnO}$ deposition on a sulfated BOPP substrate under UV light illumination $(365 \mathrm{~nm})$. The corresponding emission spectra of $\mathrm{ZnO}$ deposition layer is shown in right picture (the excitation wavelength is $325 \mathrm{~nm}$ ). Reproduced from (Yang et al, 2008). Copyright from Wiley-VCH Verlag GmbH \& Co. KGaA. Reproduced with permission.

inhibiting the $\mathrm{ZnO}$ nucleation. However, this statement is still not fully confirmed because recently Morin et al reported a reverse phenomenon that $\mathrm{COOH}$-enriched PET surface actually induced the formation of $\mathrm{ZnO}$ nanorods patterning (Morin et al, 2007).

To summarize this section, surface functional small molecule-mediated ability is demonstrated again to be used for the fabrication of positive and negative $\mathrm{ZnO}$ film on polymer substrates. Such patterned $\mathrm{ZnO}$ deposition is very sensitive to the surface functional group changes. Patterned $\mathrm{ZnO}$ films made up of vertical arrayed rods typically $500-680 \mathrm{~nm}$ in diameter, were found to form on sulfated and hydroxylated BOPP surfaces. When the substrate was changed from BOPP to polyester, the $\mathrm{ZnO}$ deposition with vertical 
$\mathrm{ZnO}$ rods typically $750 \mathrm{~nm}$ in diameter would selectively grow on unirradiated regions because of the transformation from sulfated or hydroxylated to carboxylated regions on PET substrate during the deposition process, the latter group was considered as a suppressing moiety for hindering the $\mathrm{ZnO}$ deposition. The present results gave us the $\mathrm{ZnO}$ film with microrods array. Since $\mathrm{ZnO}$ nanorods and their array have found great applications in laser and photo-energy devices, the next possible direction for this research is to optimize the reaction condition to fabricate $\mathrm{ZnO}$ nanorods array on polymer substrates by such surface functional group-mediated growth. Morin et al has presented such an approach on flexible polymer substrates. (Morin et al, 2007)

\subsection{3 $\mathrm{SiO}_{\mathrm{x}}$ film fabrication on hydroxylated polymer surfaces by biomimetic interface- directed sol-gel. (Gan et al, 2010)}

Among versatile inorganic oxides, silica oxide film as $\mathrm{SiO}_{x}$ is especially important because this semiconductor material could provide crucial properties in devices or serve as a base layer for further multilayer construction. In this section, we describe a new interfacedirected sol-gel method to fabricate flexible high quality silicon oxide film onto commodity plastics (Figure 22). Such fabrication strategy relies on CPO process described above. Firstly, $\mathrm{CPO}$ reaction was used on BOPP surface to introduce a hydroxyl monolayer. We found that this kind of hydroxyl monolayer could serve as nucleation and growth site for subsequent

\section{Whole-area deposition:}

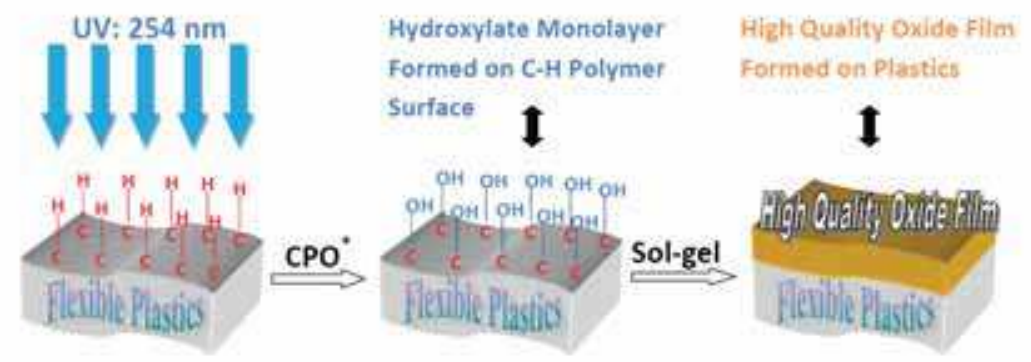

Area-selective deposition:

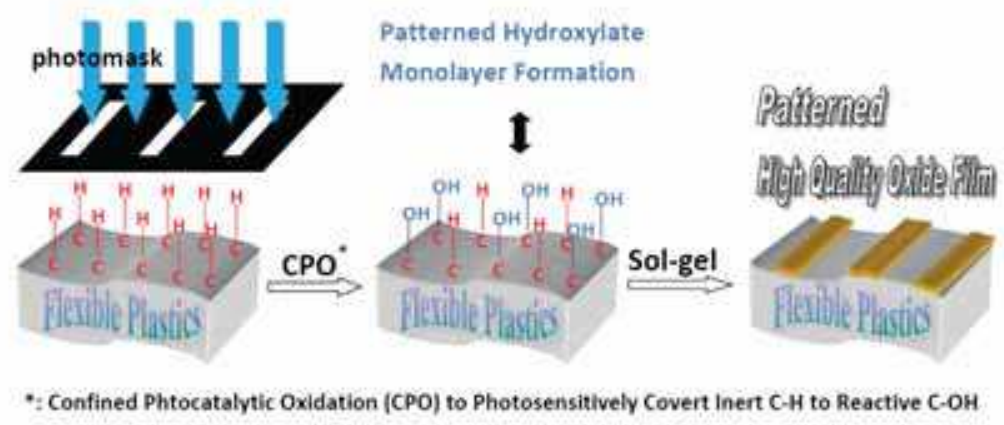

Fig. 22. The schematic process to fabricate $\mathrm{SiO}_{x}$ and its patterning on $\mathrm{BOPP}-\mathrm{OH}$ surface. Reproduced from (Yang et al, 2010). Copyright from Science in China Chemistry. Reproduced with permission. 
sol-gel reaction on the surface. Different from commonly used sol-gel technique where bulk sol-gel reaction occurs simultaneously, surface hydroxyl-directed sol-gel reaction in this work presents the unique character as surface-initiating cascade growing. Surface hydroxyl groups were capable of capturing silanol groups to initiate surface gelation. Such interface condensation reaction was possibly catalyzed by trace water and acidic molecules adsorbed onto hydrophilic hydroxyl-modified surface. The latter physisorption could become energetically favorable through the possible formation of hydrogen bond and/or protonation between trace water/acid and surface hydroxyl groups. This kind of surface condensation reaction seemed to express higher reactivity than the condensation in the Sisol, because the preferential surface growth was observed at the early deposition stage to form 4-6 nm silica layer. As a result, the deposition on the surface proceeded along a more controlled two-step way. In the first step, the deposition was initiated by the surface hydroxyl groups which could be reflected by the thin thickness data $(4-6 \mathrm{~nm})$ detected in the early stage (10-40 $\mathrm{min})$. This process would last at least $40 \mathrm{~min}$ to form enough reactive sites.
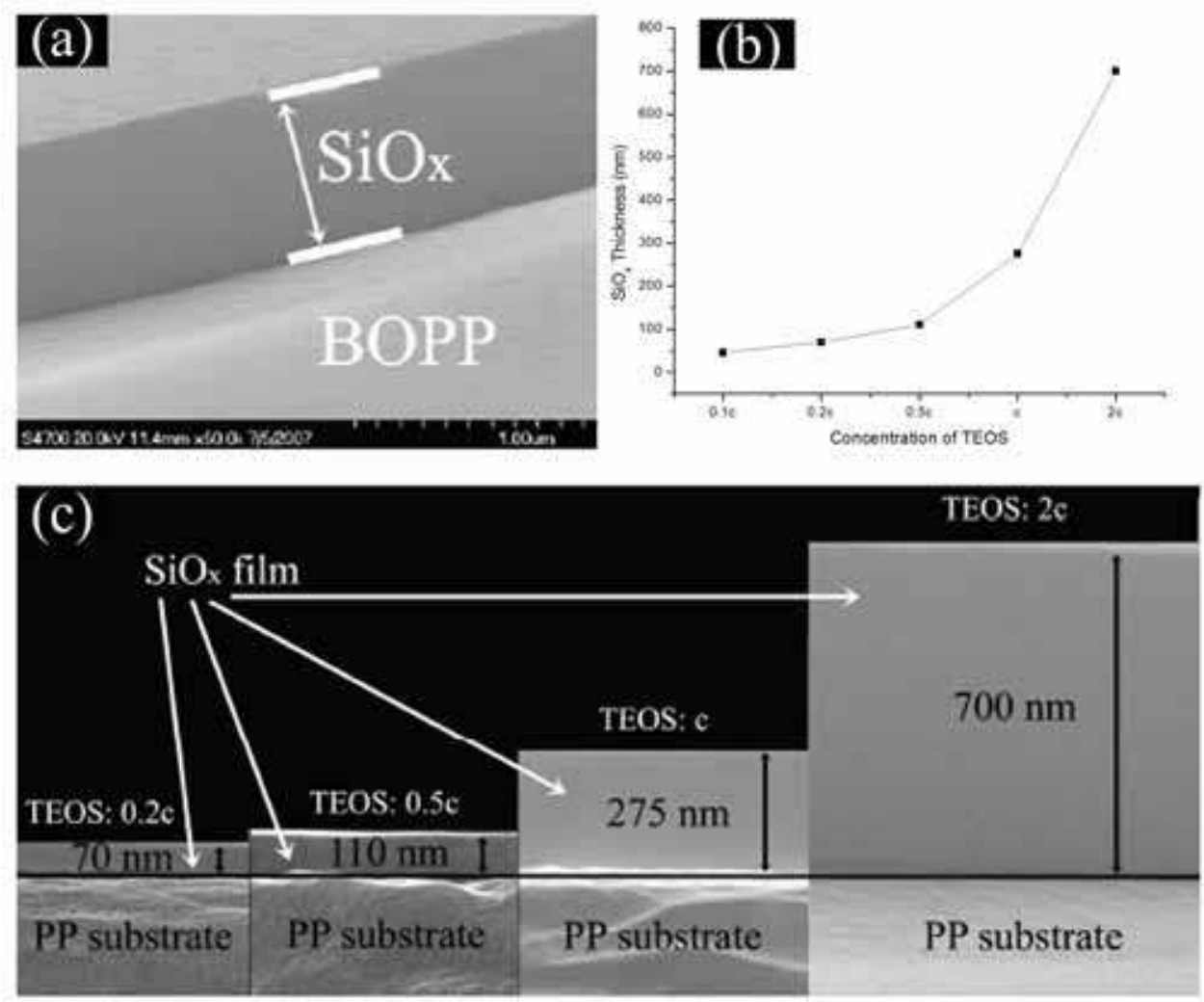

Fig. 23. A typical cross-sectional FE-SEM image (a) of $\mathrm{SiO}_{x}$ deposition on BOPP-OH surface $\left(\mathrm{BOPP}-\mathrm{OH} / \mathrm{SiO}_{\mathrm{x}}\right)$, and the controlling on the deposition thickness was facilely achieved by changing TEOS concentration, as plotted in (b) and visualized in (c). Reproduced from (Yang et al, 2010). Copyright from Science in China Chemistry. Reproduced with permission. 
After $40 \mathrm{~min}$, the mature thin silica layer served as nucleation and growth templates to gradually thicken the silica layer. Similarly, Hozumi and coworkers (Hozumi et al, 2003) also found an ultrathin SiOx layer $(\sim 1 \mathrm{~nm})$, termed as "nanoskin", could form on photosensitive polymer surface during vacuum UV enhanced vapor deposition, which facilitated further deposition of solid and ultraflat inorganic oxide.

The resulting silica was crack-free with strong covalent bonding with underlying polymer substrates, and also had homogeneous morphology with ultralow roughness, highly optical transparency, tunable thickness from $\mathrm{nm}$ to $\mu \mathrm{m}$, and easy patterning ability. As shown in Figure 23, a typical cross-sectional image of $\mathrm{BOPP}-\mathrm{OH} / \mathrm{SiO}_{\mathrm{x}}$ film indicated that a clear and even interface between $\mathrm{SiO}_{x}$ and $\mathrm{BOPP}$ phases. Homogeneous $\mathrm{SiO}_{x}$ surface also could be partially revealed from this side-view observation. Accordingly, the thickness of $\mathrm{SiO}_{x}$ layer could be directly obtained from these cross-sectional pictures. We further found that the thickness could be flexibly tuned by simply changing the sol-gel condition [e.g. tetraethylorthosilicate (TEOS) concentration and gelation time] from nanometer to micrometer, and this ability is very crucial for practical applications. The effect of TEOS concentration on the thickness increase was not a linear relationship, but a typical one observed in inorganic oxide sol-gel process.

The smooth surface morphology of resulting silica film was further revealed by AFM (Figure 24). Comparing with pristine BOPP and BOPP-OH films, the BOPP-OH/SiO surface showed very even and homogeneous morphology in large scale. The defects such as cracks, pinholes commonly observed in other methods did not appear under the observation field. Further measurement illustrated that ultra-smooth surface with extremely low root mean square (RMS) $(\sim 8 \AA)$ over large area $\left(25 \mu \mathrm{m}^{2}\right)$ was obtained after $\mathrm{SiO}_{\mathrm{x}}$ layer formed onto BOPP-OH surface. The RMS value on such ultraflat surface was lower than BOPP and BOPP-OH surfaces before the deposition and even better than that on commercial quartz surface (around $10 \AA$ ). Because the fabrication of such ultrasmooth surface only utilized the well-developed sol-gel technique and universal CPO reaction on polymer surface, this method provided us with a very simple and scalable approach to quickly fabricate ultraflat oxide film on polymeric materials. The resulting film also possessed superior optical transparence (Figure 25). Comparing with blank BOPP film, $\mathrm{BOPP}-\mathrm{OH} / \mathrm{SiO}_{x}$ film with different thickness only had very little decrease $(<3 \%)$ in the transmittance at $600 \mathrm{~nm}$, even in the case that the thickness of $\mathrm{SiO}_{\mathrm{x}}$ increased to $300 \mathrm{~nm}$. As a result, no discernible difference could be told between pristine BOPP and $\mathrm{BOPP}-\mathrm{OH} / \mathrm{SiO}_{\mathrm{x}}$ films, after direct observation by eyes on the characters ("BUCT") behind two kinds of films.
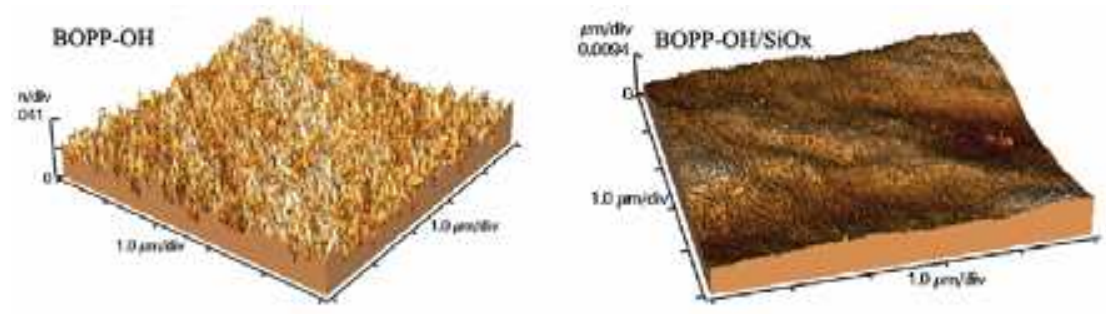

Fig. 24. Surface roughness evaluation by AFM on BOPP-OH before/after $\mathrm{SiO}_{x}$ deposition. Reproduced from (Yang et al, 2010). Copyright from Science in China Chemistry. Reproduced with permission. 


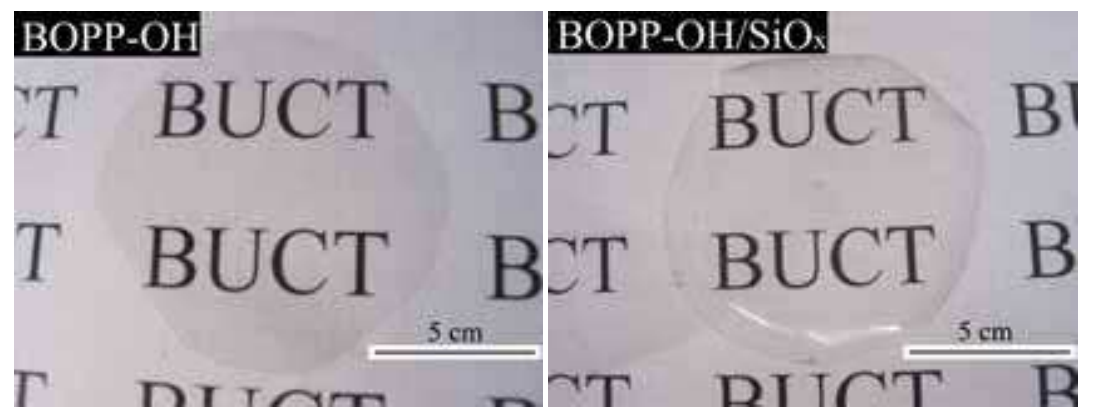

Fig. 25. The optical image after putting a $\mathrm{BOPP}-\mathrm{OH}$ or $\mathrm{BOPP}-\mathrm{OH} / \mathrm{SiO}_{x}$ film on a paper with "BUCT" characters on it. The two pictures presented the contrast between optical micrograph of BOPP-OH (left) and $\mathrm{BOPP}-\mathrm{OH} / \mathrm{SiO}_{x}$ film (right). The clear observation on underlying characters ("BUCT") proved excellent optical transparency in visible light could be obtained on BOPP-OH/ $\mathrm{SiO}_{x}$ film. Reproduced from (Yang et al, 2010). Copyright from Science in China Chemistry. Reproduced with permission.

The film could be easily patterned by applying ultrasonic or adhesive tape peeling on the asdeposited film, because the big coating adhesion difference existed between hydroxylated (covalent bonding) and unmodified regions (physisorbed). For this aim, patterned hydroxylated surface was firstly fabricated by CPO under a control of a photomask. After the same $\mathrm{SiO}_{x}$ sol-gel deposition on such patterned hydroxylation surface, the pattern was finally formed through mechanical destabilization from either ultrasonic washing or $3 \mathrm{M}$ adhesive tape peeling to remove weak deposition on unmodified regions. As shown in Figure 26, either adhesive tape peeling (left) or ultrasonic agitation (right) could give us clear circular or stripe patterns with extremely low line edge variation, presenting good copies from the photomasks used. The feature size of such patterns was also homogeneous with few defects. Moreover, a large-area homogeneous pattern $\left(\mathrm{cm}^{2}\right)$ could be also obtained with good fidelity by such a process. The cracking of inorganic layer under the deformation of organic substrates is a common problem when preparing hybrid composite film, however, no discernible cracks were observed on $\mathrm{SiO}_{x}$ layer when mechanically bending $\mathrm{BOPP}-\mathrm{OH}$ substrate because of strong interfacial bonding and low internal stress buried in $\mathrm{SiO}_{\mathrm{x}}$ layer.
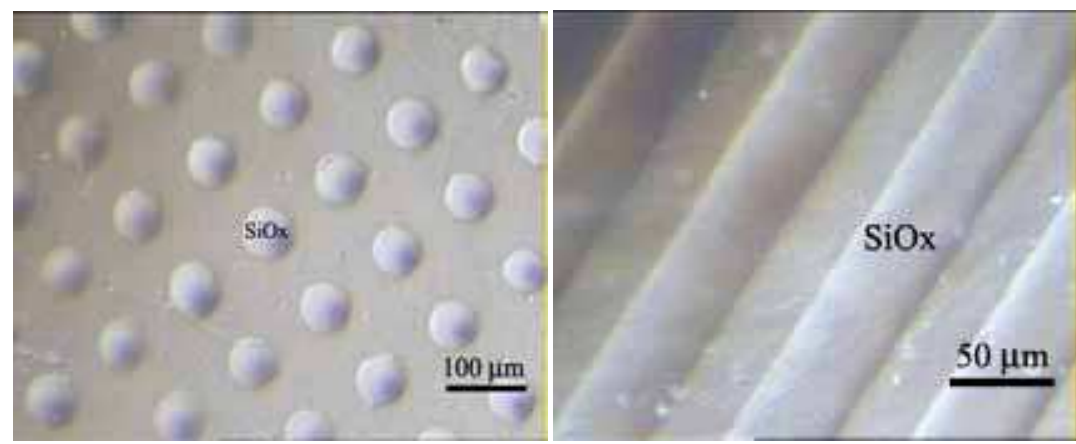

Fig. 26. Optical images of patterned $\mathrm{SiO}_{x}$ layer on $\mathrm{BOPP}-\mathrm{OH}$ surface fabricated by adhesive tape peeling (left) and ultrasonic agitation (right). Reproduced from (Yang et al, 2010). Copyright from Science in China Chemistry. Reproduced with permission. 
To summarize this section, our strategy provides a simple and effective way to fabricate ultraflat silica film on polymer substrate with strong bonding strength and easy patterning ability, excluding the requirements of clean room and vacuum devices so as to fulfill lowcost and fast fabrication demands. Future research along this direction may be suggested as follows: 1). Explore the possibility to extend this method for other oxide material fabrication. For example, we have successfully achieved $\mathrm{TiO}_{2}$ film fabrication by such method (Figure 27). 2). Explore the possibility to prepare hybrid multilayer structure by using silica film as a base layer. 3). Explore the possibility to develop the applications of this material. Herein, two application examples from such high quality $\mathrm{SiO}_{x}$ layer onto plastics are given but should not be limited within these. One is that oxygen permeation rate of $\mathrm{SiO}_{x}$ deposited polymer film decreases 25 times than pristine polymer substrate, which is good for the
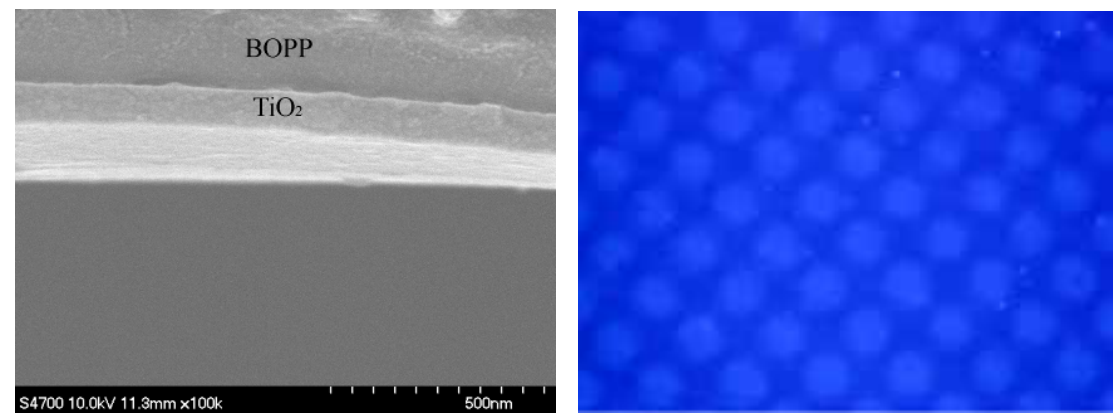

Fig. 27. Cross-sectional Field Emission (FE)-SEM image of $\mathrm{BOPP}-\mathrm{OH} / \mathrm{TiO}_{2}$ film (left) and fluorescent image (under UV excitation) of $\mathrm{TiO}_{2}$ micropatterning on $\mathrm{BOPP}-\mathrm{OH}$ surface (right) fabricated by our interface-directed sol-gel. Reproduced from (Yang et al, 2010). Copyright from Science in China Chemistry. Reproduced with permission.
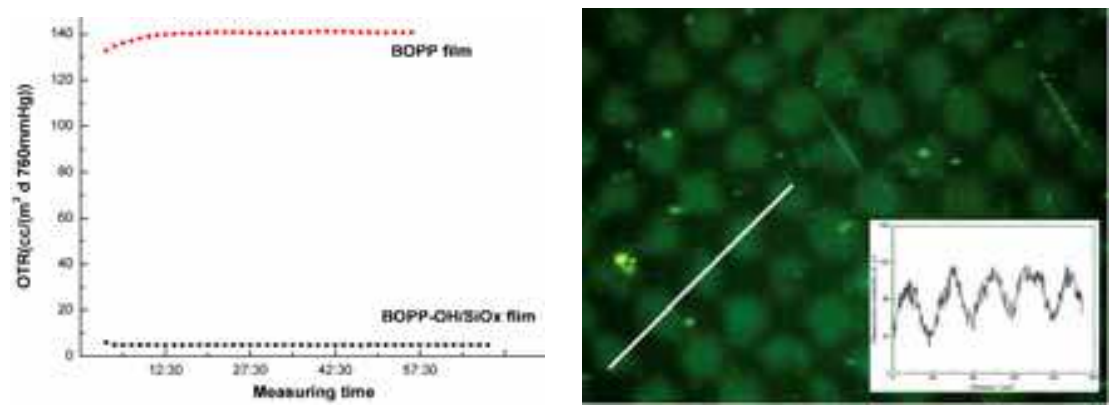

Fig. 28. Two application examples on BOPP-OH/SiO $\mathrm{S}_{x}$ samples. On the left, oxygen permeability of BOPP-OH film with only $150 \mathrm{~nm}$ thick $\mathrm{SiO}_{x}$ could get 25 times decrease than that of pristine BOPP. On the right, fluorescence microscope image demonstrated that patterned FITC-IgG immobilization could be easily achieved on patterned $\mathrm{BOPP}-\mathrm{OH} / \mathrm{SiO}_{x}$ through the decoration of amine-terminated silane, 3-aminopropyltriethoxysilane (APTES) and further activation by glutaraldehyde on the surface. The profile intensity on selected region (inset picture) showed around 3:1 signal-to-background ratio could be obtained by this type of protein immobilization. Reproduced from (Yang et al, 2010). Copyright from Science in China Chemistry. Reproduced with permission. 
potential packaging materials (Figure 28 left). The other one is that silanization monolayer, for example, 3-aminopropyltriethoxysilane (APTES), could be successfully constructed onto the resulting silica layer through classical silanization reaction, which is applicable for many potential purposes, for instance, proteins could be accordingly immobilized onto plastic support with effective signal-to-background ratio (Figure 28 right).

\subsection{Level 2: Peptide-mediated nanocrystal and superstructures formation}

Following the researches performed by small functional groups, a larger molecule, that is, inorganic-binding peptide (IBP) with oligomer grade was further used to mediate metal and inorganic material growth. IBP belongs to one kind of functional biomolecules which is capable to function as biomimetic template for material synthesis. Recently, Naik et al extenstively reviewed this field on biomolecules-mediated biomimetic material fabrication (Dickerson et al, 2008). The IBP is a short polypeptide capable to selectively reduce certain inorganic ions from solutions to form nanoparticles. In our work, four types of peptides, NPSSLFRYLPSD (AG4), AYSSGAPPMPPF (AG3), MHGKTQATSGTIQS (MS14) and DRTSTWR (PT2) have been successfully immobilized on aminated polymer surface formed by USAR method and utilized to direct the formation of shape-controlled noble metals ( $\mathrm{Pt}$, $\mathrm{Ag}$, and $\mathrm{Au}$ ) micro/nano assemblies on flexible polymer substrates. Triangle, square, sphere, and hexagon as well as superamolecular assembly structure of particles have been observed in these peptide-directed inorganic material synthesis.

Firstly, silver nanoparticles were synthesized by AG3 and AG4 peptides (Zhang et al, 2005, 2006 and 2008). Among various IBPs, AG3 (AYSSGAPPMPPF) is a special peptide sequence that specifically and selectively binds to silver (Naik et al, 2002) in a solution. Inspired by this pioneering research, we immobilized AG3 on aminated PET film surface modified by USAR. As mentioned above, the biomolecule immobilized by USAR could keep its biological activity after immobilization on the aminated surface through electrostatic coordination. After that, the substrate with AG3 immobilized was incubated directly in silver nitrate solution without any other reducing regents added. Silver crystallites with the size being 1-4 $\mu \mathrm{m}$ were found on the film surface after finishing the incubation (Figure 29). The shape of silver microcrystals was diverse including hexagonal, triangle and cubic. Hexagonal and triangular crystallites were commonly observed in such IBP-mediated biomimetic synthesis in a solution, while the cubical shape is seldom reported in the literatures so far. The possible in-plane confinement from the substrate on the nucleation and growth of the crystal may be attributed to the formation of this novel morphology. Although AG3-mediated silver synthesis is effective, the size and morphology of silver crystals are quite broad. In order to narrow the size and morphology distribution for better controlling on this synthesis, we switched from the use of AG3 to AG4, because the latter also expressed strong biomineralization effect on silver (Naik et al, 2002). After similar peptide immobilization and incubation procedures, silver microcrystals with the size being 1-2 $\mu \mathrm{m}$ were found on PET film (Figure 30). Besides these big particles, AFM scanning on the surface (Figure 31) also revealed the existence of some nanoparticles with the size being around $40 \mathrm{~nm}$ on the surface. The shapes of these crystals mainly included triangular and square. Accordingly, based on our results, it seems difficult to obtain uniform silver particles if only peptides were used in this biomimetic synthesis system.

For addressing this challenge, we developed a new approach that combines an organic matrix with IBP to perform such synthesis. This idea is actually inspired by organic matrices in some life systems which can operate as templates for biosynthesis of various materials 


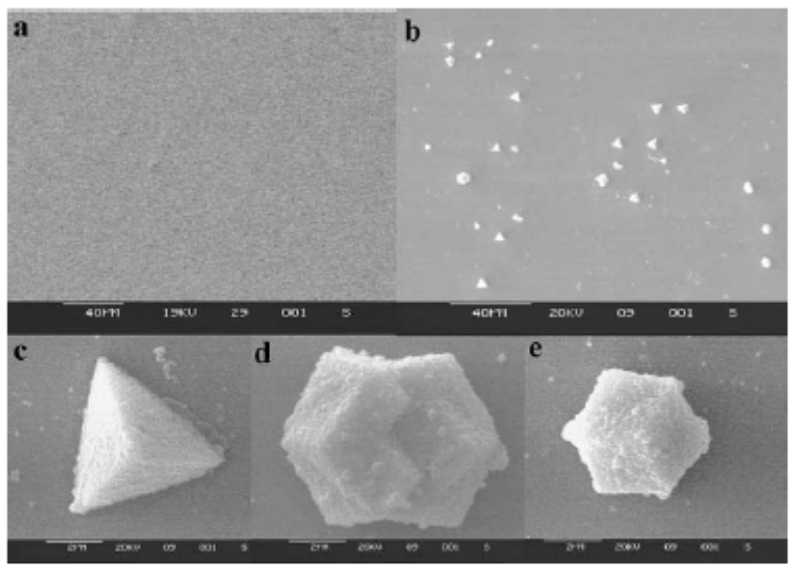

Fig. 29. Silver crystallite morphologies observed under SEM. (a) There was not any crystallite on the blank PET film (control, bar length $40 \mu \mathrm{m}$ ). (b) Various silver crystallites (white dots, bar length $40 \mu \mathrm{m}$ ). (c) Triangular silver crystallite (bar length $2 \mu \mathrm{m}$ ). (d) Hexagonal silver crystallite (bar length $2 \mu \mathrm{m}$ ). (e) Cubic lattice crystallite (bar length $2 \mu \mathrm{m}$ ). Reproduced from (Zhang et al, 2005). Copyright from Elsevier. Reproduced with permission.
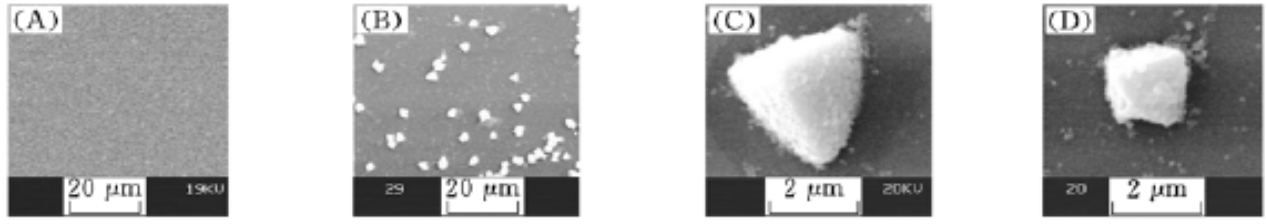

Fig. 30. SEM images of silver particles formed on (A) control (original PET without the peptide AG4 immobilized); (B). PET with AG4 immobilized. (C) and (D) are enlarged images from (B) to show two typical types of silver crystals formed in (B). Reproduced from (Zhang et al, 2006). Copyright from Chemical Journal of Chinese Universities. Reproduced with permission.
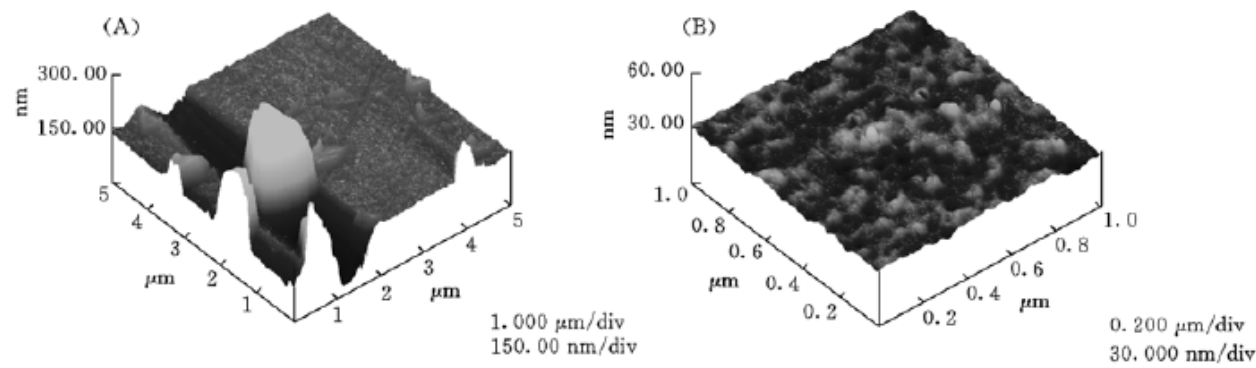

Fig. 31. AFM image on silver particle formed on PET substrate with AG4 immobilized. Reproduced from (Zhang et al, 2006). Copyright from Chemical Journal of Chinese Universities. Reproduced with permission. 
with uniform size and morphology. In our experiment, chitosan was chosen to serve as this kind of matrix. Chitosan is found to function as the main cell wall constitution of some fungi in the nature and made of 3D gridding structures from the self-organization of linear polymers. Such 3D grid texture is expected to provide good host spaces for metal ions and their in situ reduction reaction, and thereby induces the formation of uniform crystals with well controlled size and morphology (Huang et al, 2004). Based on this design, the chitosan and peptide AG4 solutions were firstly mixed well before adding silver nitrate solution. The composition in chitosan is a linear polysaccharide chain consisting of randomly distributed copolymer from D-glucosamine and N-acetyl-D-glucosamine. Therefore, abundant amines along chitosan chain are considered to form coordination bonding with silver ions, which can serve as nucleation sites in the reducing environment provided by AG4. As shown in Figure 29, the resulting silver crystals expressed low polydispersity on morphology and size. This control from chitosan was concentration-dependent. When the concentration of chitosan was in the range of $(0.01 \%-0.1 \%)$, the size change of silver nanoparticles was not obvious, with nanoparticles being about $20 \mathrm{~nm}$ in average size (Figure 32a, b). With further increase of chitosan concentration above $0.1 \%$, the size of silver nanoparticles became smaller with the diameter being around $5 \mathrm{~nm}$ (Figure 32c, d). When the chitosan concentration is changed, the host space volume and nucleation sites for silver growth is correspondingly changed, which could be responsible for the size change of the silver nanoparticles.

Besides silver, we also extended this surface-immobilized IBP-based reaction to other peptide system for other material synthesis. For example, the polypeptide sequence MS14 (MHGKTQATSGTIQS) (Brown et al, 1997 and 2001; Braun et al, 2002; Kulp et al, 2004; Tamerler et al, 2006) was used for biomimetic preparation of gold nanoparticles and their aggregates (Wang et al, 2007). As shown in Figure 33, MS14-mediated reduction of $\mathrm{HAuCl}_{4}$ on PET surface resulted in single-crystallite formed on the surface, presenting hexagonal, triangular and quadrangular morphology with 1-3 $\mu \mathrm{m}$ in size. There were also small spherical particles, approximately $200 \mathrm{~nm}$ in size found on the same substrate. Further effort to combine organic matrix with MS14 for controllable preparation of uniform gold nanoparticles could be suggested as the next future work. After successful preparation of gold and silver crystals, another kind of important noble metal, platinum crystals were also prepared by the peptide PT2(DRTSTWR) (Sarikaya et al, 2003) immobilized on PET surface. Platinum microparticles with the size being 1-2 $\mu \mathrm{m}$ were observed on PT2-functionalized PET surface (Li et al, 2007).

To summarize this section, surface-immobilized peptides have expressed certain potential to in situ reduce and fabricate metal nanocrystals on the film surface. However, some questions and problems must be addressed: 1). Exact biomimetic reaction mechanism must be elucidated; 2). The size and morphology of the resulting crystals must be well controlled. Some organic compounds such as chitosan, PAA have showed certain potential for this controlling ability. In this aspect, we actually found that the size and morphology of particles obtained by surface-immobilized peptide was often much wider than that of particles obtained by same peptide but freely dissolved in a solution. For example, in the case that the synthesis of Pt crystals, instead of the use of surface immobilized PT2, simple use of a mixture solution of free PT2 and $\mathrm{PtCl}_{4}$ could give us much smaller Pt nanoparticles ranging from $1.5-2 \mathrm{~nm}$. The question that whether the surface immobilization process would affect the biological mediation ability of peptides and nucleation as well as growth process of crystals needs to be clearly anwered. 

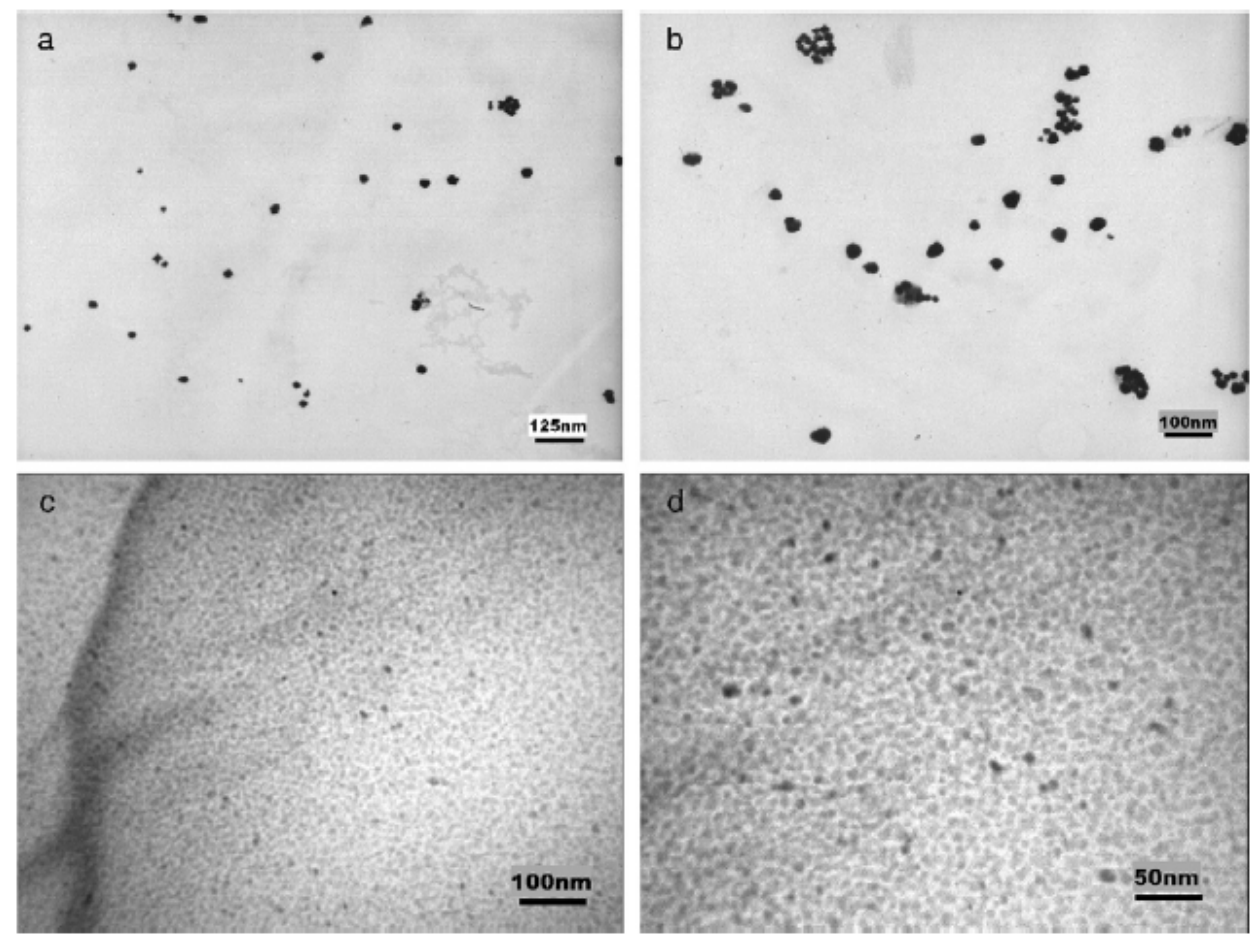

Fig. 32. Transmission Electron Microscope (TEM) images of silver nanoparticles prepared with various chitosan concentrations (wt.\%) without adjusting silver ion concentration. (a) $0.05 \%$; (b) $0.1 \%$; (c) $0.5 \%$; (d) $1 \%$. Reproduced from (Zhang et al, 2008). Copyright from Elsevier. Reproduced with permission.

\subsection{Level 3: Polymer brush-mediated quantum dot formation (Zou et al, 2009)}

Finally, we reached the macromolecular grade for this kind of research. In this aspect, we utilized photo-initiated surface grafting systems to construct large-area functional acidic polymer brush, PAA on arbitrary polymer substrate, and further test for inorganic material growth showed that surface-immobilized polymer brush was able to mediate the formation of $\mathrm{ZnO}$ quantum dots (QD) (Figure 34) and patterning within polymer brush regions. To our best of knowledge, before our report, the formation of $\mathrm{ZnO}$ quantum dot mediated by surface-immobilized polymer brush is never reported. In our experiment, a typical two-step biomimetic procedure was employed: firstly, the film with PAA brush (BOPP-g-PAA) was soaked in zinc chloride solution and achieved a saturated complexation between zinc ions and carboxyl groups in PAA chains. After that, the film with zinc complexed was incubated in HMT solution under suitable temperature and time, and finally, ZnO QDs were in situ formed within PAA brush on BOPP surface, resulting in an organic/inorganic hybrid nanocomposite film (BOPP-g-PAA/ $\mathrm{ZnO}$ ).

As shown in Figure 35, FE-TEM directly demonstrated the formation of ZnO QD after incubating polymer brush-grafted film in HMT solution. The size of these nanoparticles ranging from $4-5 \mathrm{~nm}$ was in the regime of quantum size of $\mathrm{ZnO}$. For single $\mathrm{ZnO} \mathrm{QD}$, a 


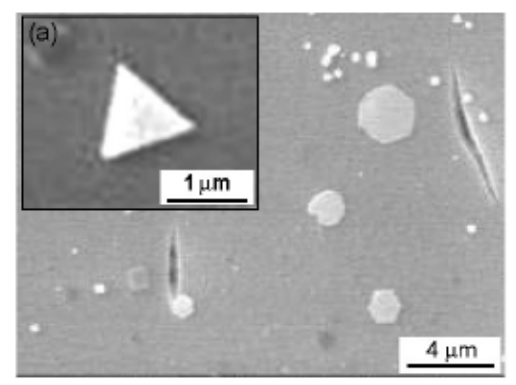

(b)
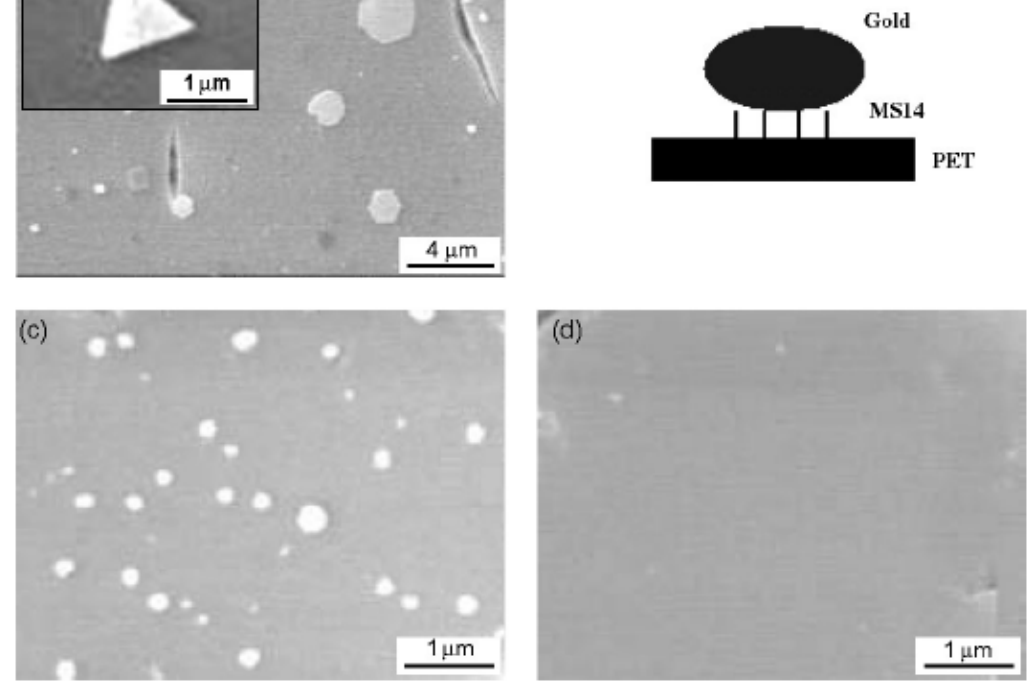

Fig. 33. SEM image and schematic illustration. (a) Various (including triangular, hexagonal and cubic lattice) single crystallites formed on PET and not moved away with $\mathrm{ddH}_{2} \mathrm{O}$. (b) Schematic illustration of (a). (c) Spherical particles deposited on blank PET film. (d) Control: there were no particles on the blank PET film after washing with $\mathrm{ddH}_{2} \mathrm{O}$. Reproduced from (Wang et al, 2007). Copyright from Wiley-VCH Verlag GmbH \& Co. KGaA. Reproduced with permission.

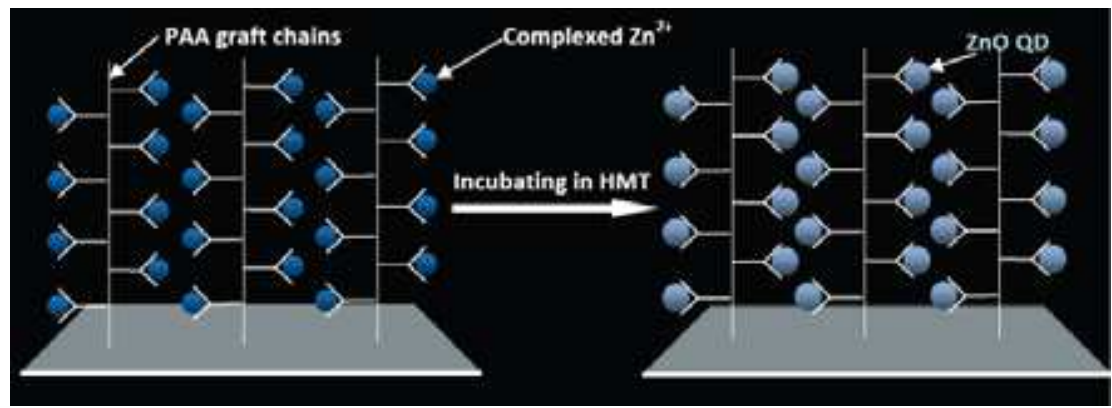

Fig. 34. The schematic process of $\mathrm{ZnO}$ QD formation mediated by surface immobilized PAA brushes. PAA chains grafted surface (white tree-like structures) was soaked in $\mathrm{Zn}\left(\mathrm{NO}_{3}\right)_{2}$ aqueous solution $(0.1 \mathrm{M})$ for $1 \mathrm{hr}$ to achieve the complex between carboxylic groups (from grafted PAA chains) and $\mathrm{Zn}^{2+}$ (symbolized by dark blue spheres). After washing, the surface with complexed $\mathrm{Zn}^{2+}$ was incubated in HMT aqueous solution $(0.1 \mathrm{M})$ for 2 days at $90^{\circ} \mathrm{C}$. HMT provided $\mathrm{OH}^{-}$source for the hydrolysis and precipitation of $\mathrm{ZnO}$ to transform complexed $\mathrm{Zn}^{2+}$ to $\mathrm{ZnO}$ QD (light blue spheres). Reproduced from (Zou et al, 2009). Copyright from Wiley-VCH Verlag $\mathrm{GmbH} \& \mathrm{Co}$. KGaA. Reproduced with permission. 

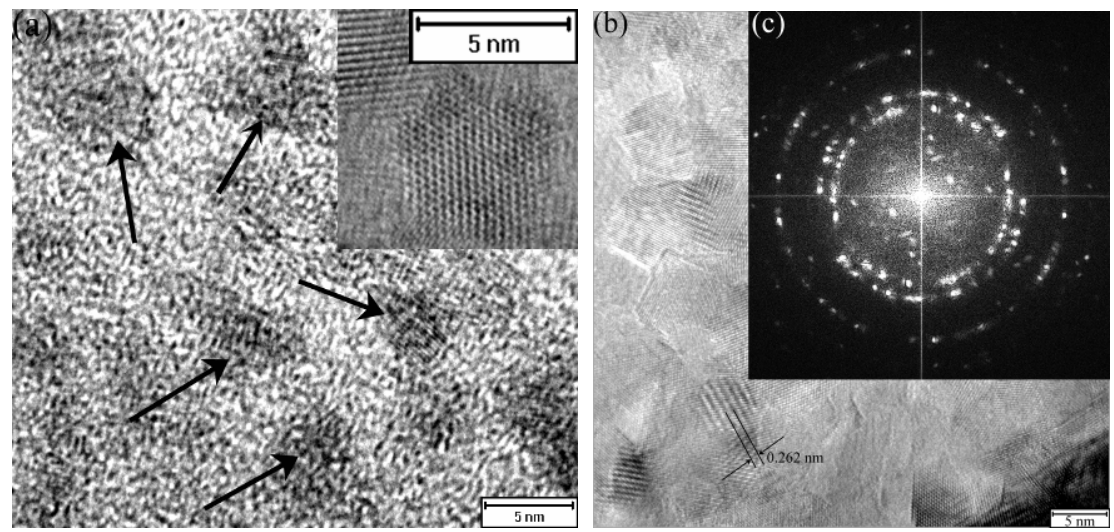

Fig. 35. FE-TEM, High-resolution (HR)-TEM images (a, b), and SAED pattern (c) of ZnO QD obtained by our method. Inset picture in (a) showed clearly an enlarged ZnO QD with hexagonal wurtize-type lattice. Reproduced from (Zou et al, 2009). Copyright from Wiley$\mathrm{VCH}$ Verlag $\mathrm{GmbH} \& \mathrm{Co}$. KGaA. Reproduced with permission.
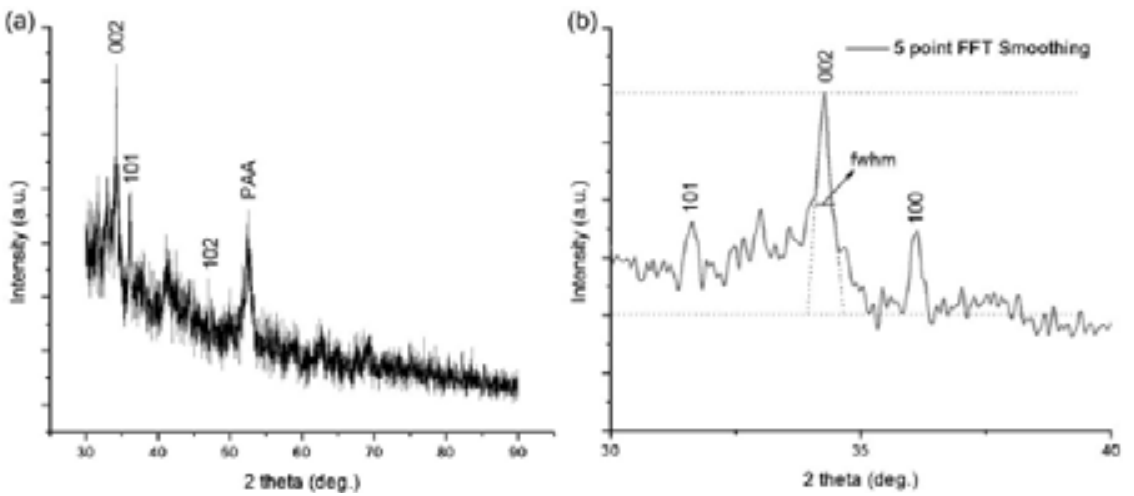

Fig. 36. (a) XRD pattern of BOPP-g-PAA/ZnO QD nanocomposite film and (b) the corresponding fitting curve by 5-point FFT smoothing. The (002), (101) and (102) diffraction patterns could be matched with the standard pattern of a wurtzite $\mathrm{ZnO}$ crystal from the JCPDS database. A preferred orientation was observed to be the (002) orientation. The peak at 528 is ascribed to organic component (PAA). Reproduced from (Zou et al, 2009).

Copyright from Wiley-VCH Verlag $\mathrm{GmbH} \& \mathrm{Co}$. KGaA. Reproduced with permission.

uniform hexagonal lattice structure was observed, which signified a single crystal nature without defects. This single crystal nature was also supported by the dotted Selected Area Electron Diffraction (SAED) pattern. (002) direction was found to be the brightest spots in SAED pattern, which revealed that the preferential growth direction for this $\mathrm{ZnO}$ QD was (002) direction (c-axis). The spacing distance between two diffraction planes in single QD was estimated as $0.262 \mathrm{~nm}$ from high-resolution(HR)-TEM image, this value was a typical $\mathrm{d}$ spacing of the (002) planes in a wurtize-type $\mathrm{ZnO}$. This wurtize type was further supported by XRD pattern of BOPP-g-PAA/ZnO film (Figure 36), where three characteristic diffraction 

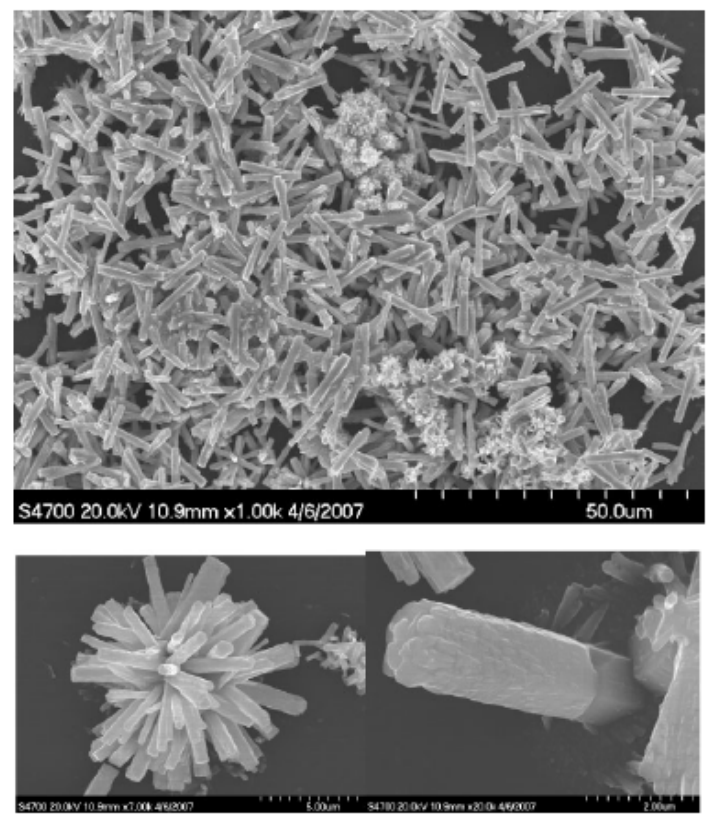

Fig. 37. ZnO particles grew on the surface without PAA biomimetic mediation. BOPP-gPAA film was directly placed into the mixed solution of $\mathrm{Zn}\left(\mathrm{NO}_{3}\right)_{2}$ and $\mathrm{HMT}(0.1 \mathrm{M})$. The growth took place at $90^{\circ} \mathrm{C}$ for $48 \mathrm{~h}$. The lower two images are the amplification of the upper image to show two typical kinds of microcrystallites (flowers and rods), which are found on such a surface. Reproduced from (Zou et al, 2009). Copyright from Wiley-VCH Verlag $\mathrm{GmbH} \& \mathrm{Co}$. KGaA. Reproduced with permission.

peaks at (002), (101) and (102) were observed. The highest intensity at (002) plane reflected again that the resulting $\mathrm{ZnO}$ QD had dominated c-axis direction.

It should be noted that the above-mentioned two-step biomimetic procedure was extremely necessary for the formation of ZnO QDs. As a control experiment, we deliberately used the same PAA grafts without pre-doping of $\mathrm{Zn}^{2+}$ for incubation in a mixture solution of zinc chloride and HMT. We found that in such a system, the mediation ability of PAA brush was diminished and only large rod-like microcrystallines on the underlying film were obtained (Figure 37). Therefore, we inferred that the effective separation of heterogeneous nucleation and growing process by two-step protocol in our method gave enough time and space to PAA, and thereby the coordination and mediation ability of PAA brushes could be fully reserved. In nature, biofabrication in organisms also often utilize this kind of process where carboxylate groups from biomolecules induce minerals formation within organic matrix through interacting with mineral ions (Estroff, 2008).

The resulting hybrid material from $\mathrm{ZnO} \mathrm{QD}$ and PAA expressed interesting photoluminescence property. Two excitation peaks at 323 and $339 \mathrm{~nm}$ in the spectrum of BOPP-g-PAA/ZnO film were observed (Figure 38a). These excitation peaks took an obvious blue shift compared with the band gap of bulk ZnO crystal at $365 \mathrm{~nm}$ (Abdullah et al, 2003; Mikrajuddin et al, 2002; Dijken et al, 2000; Brus, 1986), indicating the quantum effect of ZnO particles in the composite film. By using $325 \mathrm{~nm}$ as excitation light, we further investigated 
emission spectra of this composite film. An intensive emission in the range of 350-500 nm was found for the composite film, which was 5-fold higher than those from blank BOPP and BOPP-g-PAA films (Figure 38b). A slight split in the present emission was also observed at the near UV range as 380 and $400 \mathrm{~nm}$. Bound-excitation and vacancy-related transitions are responsible for the UV emission around $390 \mathrm{~nm}$ (Huang et al, 2001; Saito et al, 2002), and the splitting could be attributed to a consequence of the interfacial interaction of $\mathrm{ZnO}$ nanoparticles with the surrounding PAA material (Vollath et al, 2004). This interaction is facilitated by the formation of ester like linkage: $-(\mathrm{C}=\mathrm{O})-\mathrm{O}-(\mathrm{ZnO})$ (Ravindran et al, 2005; Wu et al, 1997; $\mathrm{Hu}$ et al, 1991). Our XPS data also gave the evidence for the formation of this kind of ester like linkage. Such ester like linkage could facilitate highly efficient electron
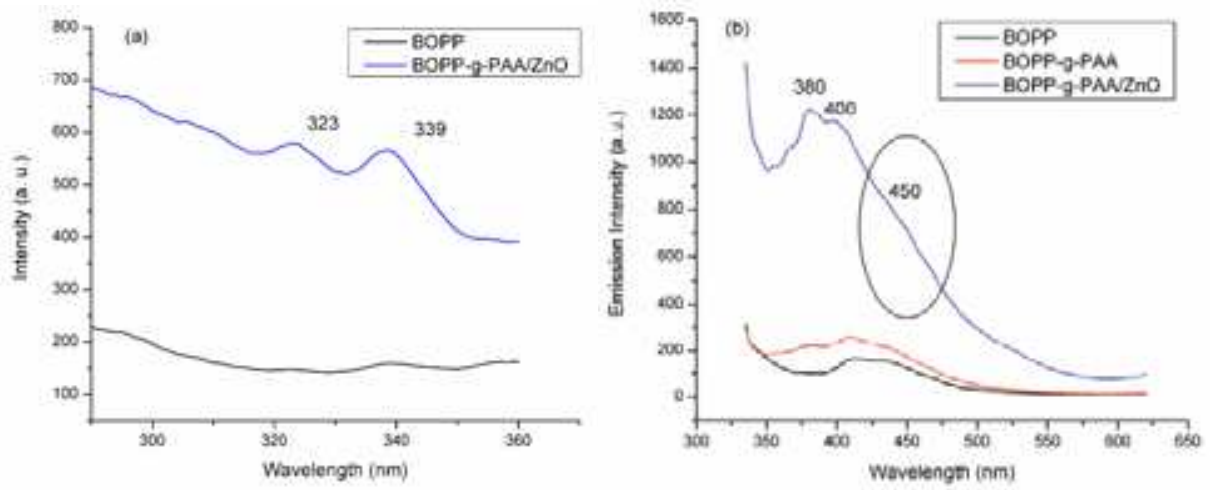

Fig. 38. Excitation (a) and emission spectra (b) of several films. In excitation spectra, the emission wavelength was settled as $385 \mathrm{~nm}$. For BOPP-g-PAA/ZnO, two maximum peaks are at about 323 and $339 \mathrm{~nm}$. In emission spectra, excitation wavelength was $325 \mathrm{~nm}$. Three much higher maximum peaks at about $380 \mathrm{~nm}, 400 \mathrm{~nm}$ and unconspicuous shoulder at 450 $\mathrm{nm}$ were found in the curve for BOPP-g-PAA/ $\mathrm{ZnO}$ composite film. Reproduced from (Zou et al, 2009). Copyright from Wiley-VCH Verlag GmbH \& Co. KGaA. Reproduced with permission.

transportation, resulting in the split on the emission peak. We also noticed that defectrelated transition usually observed at long wavelength like $600 \mathrm{~nm}$ was not observed in our composite film, indicating ZnO QD in our nanocomposite film had high quality with few defects. This judgment was also consistent with HR-TEM observations (Figure 35). The slight shoulder peak at $450 \mathrm{~nm}$ implied that such composite film had the ability for blue light emission devices (Figure 39).

Our hybrid nanocomposite film also possessed excellent optical transparency (Figure 40). Comparing with pristine and BOPP-g-PAA films, the introduction of ZnO QDs in PAA just got a little decrease in the transmittance percent. Actually, high transmittance percent (about $90 \%$ ) in the wavelength between 400 to $900 \mathrm{~nm}$ could be obtained on BOPP-g-PAA/ZnO films. This reason why high transmittance could be kept after $\mathrm{ZnO}$ QD introduced was because of a homogeneous dispersion of $\mathrm{ZnO}$ QDs in the polymer matrix with a mean diameter of no more than $5 \mathrm{~nm}$ (as revealed by TEM), therefore possible light scattering and diffraction from large particles and aggregates was effectively eliminated. This highly optical transparence is very important for advanced opto-electrical devices. Another 
important requirement for opto-electrical device is the ability to large scale patterning/array of functional nanocomposite film. This is easily achieved by our method because of the use of photo-initiated polymerization. A photomask which can effectively control irradiation path on the surface was simply applied during the photografting polymerization of AA on the surface. As a result, patterned PAA polymer brush could be obtained (Wang et al, 2005) and then region-selective fabrication of the above $\mathrm{ZnO} Q \mathrm{QD} / \mathrm{PAA}$ nanocomposite hybrid could be easily achieved. As shown in Figure 41, the pattern of PAA/ZnO QD composite film could form on the underlying substrate in a large area $\left(5 \mathrm{~mm}^{2}\right)$, which is especially appealing in macroelectronics (Morin et al, 2007; Forrest, 2004; Henzie et al, 2006; Reuss et al, 2006). The circular nanocomposite film array distributed evenly across the surface with few defects and good size homogenous. The thickness of the composite film was revealed as $2.8 \mu \mathrm{m}$ by AFM profile analysis, only a little higher than pure PAA film before ZnO incorporation $(2.5 \mu \mathrm{m})$. This fact reflected that instead of simply adding upon PAA layer, the newly formed $\mathrm{ZnO}$ QDs mainly filled into the free spaces inside PAA film, because simple adding upon PAA layer would result in an obvious thickness increase.

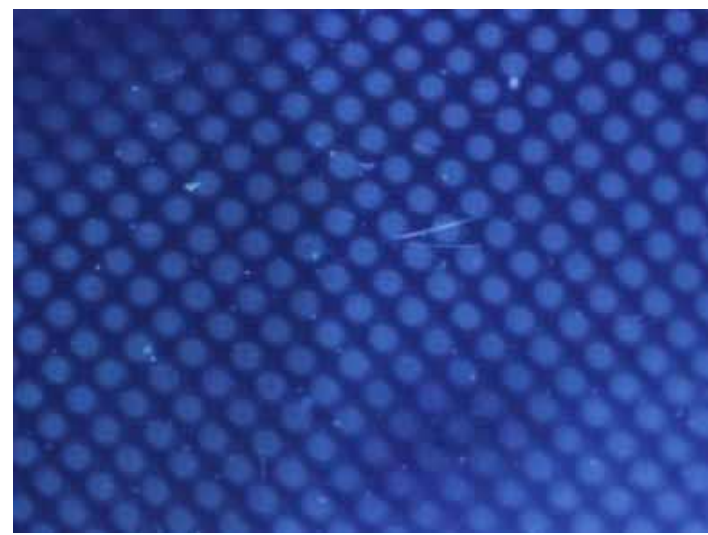

Fig. 39. The fluorescence microscopy image of patterned PAA/ZnO QD arrays on BOPP surface. Using UV exciting light, every circle area as PAA/ZnO QD hybrid represents a composite region emitting blue light. Reproduced from (Zou et al, 2009). Copyright from Wiley-VCH Verlag GmbH \& Co. KGaA. Reproduced with permission.

We also found that PAA was not an exclusive template for this biomimetic fabrication. When PAA was replaced by other similar acidic polymers with the same carboxyl groups, similar enhanced fluorescent emission was observed. In contrast, control experiments showed that switching the use of polymer brush from these carboxyl-contained acidic polymers to ester polymers (i.e. deactivation of carboxyl to ester) would result in very weak fluorescent enhancement or even lowered fluorescent emission compared with pristine polymer films before $\mathrm{ZnO}$ introducing. Such contrast strongly implies that carboxyl group plays a key role for the formation of $\mathrm{ZnO}$ QD in our biomimetic synthesis design, and any acidic polymers having carboxyl groups could become candidates for this synthesis. This finding agrees with the common accepted assumption that carboxyl group (and other functional groups)-based interaction on inorganic surface would be basic and central molecular tailoring mode in biomineralization process. 


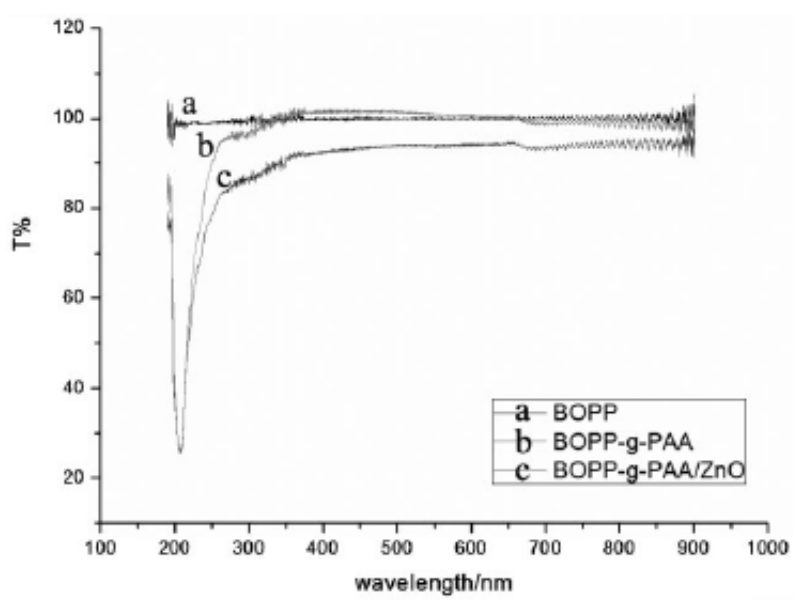

Fig. 40. The UV-vis transmittance spectra of three samples: BOPP (a), BOPP-g-PAA (b) and BOPP-g-PAA/ZnO QD (c). The thickness of BOPP, BOPP-g-PAA and BOPP-g-PAA/ZnO is around 25.7, 28.2, 28.5mm respectively. Reproduced from (Zou et al, 2009). Copyright from Wiley-VCH Verlag GmbH \& Co. KGaA. Reproduced with permission.
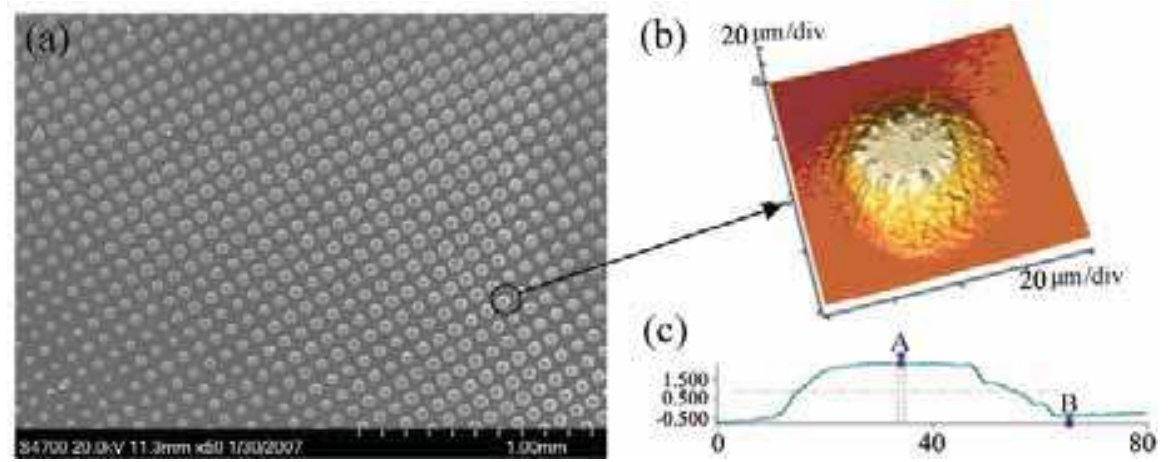

Fig. 41. FE-SEM micrograph (a) and AFM profile (b, c) of patterned PAA/ZnO arrays on BOPP surface. Reproduced from (Zou et al, 2009). Copyright from Wiley-VCH Verlag $\mathrm{GmbH} \& \mathrm{Co}$. KGaA. Reproduced with permission.

In conclusion, our results finally demonstrated that polymer brush grown on polymer substrate for example PAA used in this work had great potential to fabricate ZnO QD by a biomimetic chemical approach. The resulting ZnO QD was in situ embedded into acidic polymer templates, giving rise to the formation of a hybrid nanocomposite film. Such mediation ability came from surface photografted acidic polymer where the complex interaction between carboxyl groups in PAA and $\mathrm{Zn}^{2+}$ played a key role. Because the $\mathrm{ZnO}$ QD was homogeneously dispersed in polymer matrix, the final polymer/ZnO QD nanocomposite was a highly transparent inorganic/organic hybrid film which could be constructed on arbitrary inorganic and organic substrates. Patterning of such nanocomposite film could be also very easily achieved by simply applying a photomask in this 
photopolymerization process. The resulting patternable array of nanocomposite film had good quality and large area. Possible next work along this direction may be suggested to explore other kinds of polymer brushes for biomimetic material fabrication, since the field about polymer brush-mediated biomineralization, especially non-calcium material biomineralization is still in the early developing stage.

\section{Summary and outlook}

All of the above research results could produce inorganic and metal oxide micropatterning as well as organic/inorganic hybrid film array on soft matter surface, which would contribute to the current fast development of flexible polymer-based biomedical, energetical and optical sensors. We believe that our systematical explorations on non-calcium inorganic material fabrication mediated by surface-immobilized organic molecular template could find its potential in the fundamental and engineering researches on polymer-supported advanced technologies, biomineralization, inorganic nanocrystals and macroelectronics realms. Obviously, the future development direction in this field should be located at extensive investigation and engineering of the mediation ability of various functional groups, polypeptide, polymer brush immobilized on surfaces for the inorganic and metal materials fabrication and patterning achieving.

\section{References}

Abdullah, M.; Lenggoro, I. W.; Okuyama, K.; Shi, F. G. (2003). In Situ Synthesis of Polymer Nanocomposite Electrolytes Emitting a High Luminescence with a Tunable Wavelength. J. Phys. Chem. B 107, 1957-1961, ISSN 1089-5647.

Brown, S. (1997). Metal-recognition by repeating polypeptides. Nat. Biotechnol. 15, 269-272.

Brown, S. (2001). Protein-mediated particle assembly. Nano Lett. 1, 391-394, ISSN 1530-6992.

Brus, L. (1986). Electronic wave functions in semiconductor clusters: experiment and theory. J. Phys. Chem. 90, 2555-2560, ISSN 0022-3654.

Bamford, C. H.; AILamee, G. K. (1994). Studies in polymer surface functionalization and grafting for biomedical and other applications. Polymer 35, 2844-2852, ISSN 00323861.

Bruice, T. C.; Fife, T. H. (1961). The nature of neighboring hydroxyl group assistance in the alkaline hydrolysis of the ester bond. Tetrahedron Lett. 2, 8, 263-266, ISSN 0040-4039.

Bruice, T. C.; Fife, T. H. (1962). Hydroxyl Group Catalysis. III. ${ }^{1}$ The Nature of Neighboring Hydroxyl Group Assistance in the Alkaline Hydrolysis of the Ester Bond. J. Am. Chem. Soc. 84, 1973-1979, ISSN 0002-7863.

Braun, R.; Sarikaya, M.; Schulten, K. (2002). Genetically engineered gold-binding polypeptides: Structure prediction and molecular dynamics. J. Biomater. Sci. Polym. Ed. 13, 747-757, ISSN 0920-5063.

Chaudhary, S.; Kim, H. J.; Singh, K. V.; Ozkan, M. (2004). Fluorescence Microscopy Visualization of Single-Walled Carbon Nanotubes Using Semiconductor Nanocrystals. Nano Lett. 4, 2415-2419, ISSN 1530-6992.

Coffman, E. A.; Melechko, A. V.; Allison, D. P.; Simpson, M. L.; Doktycz, M. J. (2004). Surface Patterning of Silica Nanostructures Using Bio-Inspired Templates and 
Directed Synthesis. Langmuir 20, 20, 8431-8436, ISSN 0743-7463 (print), 1520-5827 (online)

Crookes-Goodson, W.; Slocik, J. J. M.; Naik, R. R. (2008). Bio-directed Synthesis and Assembly of Nanomaterials. Chem. Soc. Rev. 37, 11, 2403-2412, ISSN 0306-0012 (print), 1460-4744 (online)

Dutschke, A.; Diegelmann, C.; Löbmann, P. (2003). Nucleation and Growth of $\mathrm{TiO}_{2}$ Thin Films on Modified Polystyrene Surfaces. Chem. Mater. 15, 3501-3506, ISSN 08974756 .

Dickerson, M. B.; Sandhage, K. H.; Naik, R. R. (2008). Protein- and Peptide-Directed Syntheses of Inorganic Materials. Chem. Rev. 108, 4935-4978, ISSN 0009-2665.

van Dijken, A.; Meulenkamp, E. A.; Vanmaekelbergh, D.; Meijerink, A. (2000). Identification of the transition responsible for the visible emission in $\mathrm{ZnO}$ using quantum size effects. J. Lumin. 90, 123, ISSN 0022-2313.

Estroff, L. A. (2008). Introduction: Biomineralization. Chem. Rev. 11, 4329-4331. This is a speical issue of Chemical Reviews, including 23 papers ranging from pp. 4329 to pp. 4978. ISSN 0009-2665.

Forrest, S. R. (2004). The path to ubiquitous and low-cost organic electronic appliances on plastic. Nature 428, 911-918, ISSN 0028-0836.

Gan, S. H.; Yang, P.; Yang, W. T. (2010). Interface-directed Sol-gel: Direct fabrication of the Covalently Attached Ultraflat Inorganic Oxide Pattern on Functionalized Plastics. Sci. China-Chem. 53, 1, 173-182, ISSN 1674-7291 (print), 1862-2771 (online)

Gan, S. H.; Yang, P.; Yang, W. T. (2009). Photoactivation of Alkyl C-H and Silanization: A Simple and General Route to Prepare High-Density Primary Amines on Inert Polymer Surfaces for Protein Immobilization. Biomacromolecules 10, 5, 1238-1243, ISSN 1525-7797.

House, D. A. (1962). Kinetics and Mechanism of Oxidations by Peroxydisulfate. Chem. Rev. 62, 185-203, ISSN 0009-2665.

Henzie, J.; Barton, J. E.; Stender, C. L.; Odom, T. W. (2006). Large-area nanoscale patterning: Chemistry meets fabrication. Acc. Chem. Res. 39, 249-257, ISSN 0001-4842.

Huang, M. H.; Mao, S.; Feick, H.; Yan, H.; Wu, Y.; Kind, H.; Weber, E.; Russo, R.; Yang, P. (2001). Room-Temperature Ultraviolet Nanowire Nanolasers. Science 292, 18971899, ISSN 1095-9203.

Hozumi, A.; Masuda, T.; Sugimura, H.; Kameyama, T. (2003). Oxide Nanoskin Formed on Poly(methyl methacrylate). Langmuir 19, 18, 7573-7579, ISSN 0743-7463.

Hu, H.; Saniger, J.; Garcia-Alejandre, J.; Castaňo, V. M. (1991). Fourier transform infrared spectroscopy studies of the reaction between polyacrylic acid and metal oxides. Mater. Lett. 12, 281-285, ISSN 0167-577X.

Hsu, J. W. P.; Tian, Z. R.; Simmons, N. C.; Matzke, C. M.; Voigt, J. A.; Liu, J. (2005). Directed spatial organization of zinc oxide nanorods. Nano Lett. 5, 83-86, ISSN 1530-6992.

Huang, H. Z.; Yuan, Q.; Yang, X. (2004). Preparation and characterization of metal-chitosan nanocomposites. Colloids Surf., B Biointerfaces 39, 31-37, ISSN 0927-7765.

Karayannidis, G. P.; Achilias, D. S. (2007). Chemical recycling of poly(ethylene terephthalate). Macromol. Mater. Eng. 292, 128-146, ISSN 1438-7492 (print), 14392054 (online). 
Kumar, S.; Ito, T.; Yanagihara, Y.; Oaki, Y.; Nishimura, T.; Kato, T. (2010). Crystallization of Unidirectionally Oriented Fibrous Calcium Carbonate on Thermo-Responsive Polymer Brush Matrices. CrystEngComm 12, 2021-2024, ISSN 1466-8033.

Kang, J. S.; Kang, H. S.; Pang, S. S.; Shim, E. S.; Lee, S. Y. (2003). Investigation on the origin of green luminescence from laser-ablated $\mathrm{ZnO}$ thin film. Thin Solid Films 443, 1, 5-8, ISSN 0040-6090.

Kim, D. J.; Lee, K. B.; Chi, Y. S.; Kim, W. J.; Paik, H. J.; Choi, I. S. (2004). Biomimetic Formation of Silica Thin Films by Surface-Initiated Polymerization of 2(Dimethylamino)ethyl Methacrylate and Silicic Acid. Langmuir, 20, 19, 7904-7906, ISSN 0743-7463 (print), 1520-5827 (online)

Kim, D. J.; Lee, K. B.; Lee, T. G.; Shon, H. K.; Kim, W. J.; Paik, H. J.; Choi, I. S. (2005). Biomimetic Micropatterning of Silica by Surface-Initiated Polymerization and Microcontact Printing. Small 1, 10, 992 - 996, ISSN 1613-6810 (print), 1613-6829 (online)

Kulp, III J. L.; Sarikaya, M.; Evans, J. S. (2004). Molecular characterization of a prokaryotic polypeptide sequence that catalyzes Au crystal formation. J. Mater. Chem. 14, 23252332, ISSN 0959-9428 (print), 1364-5501 (online).

Li. F.; Wang, Z.; Chen, J. C.; Yang, W. T. (2007). Exploiting Preparation of Platinum Nanocrystals with Peptide PT2 (DRTSTWR). Acta Chimica Sinica 65, 22, 2644-2648, ISSN 0567-7351

Morin, S. A.; Amos, F. F.; Jin, S. (2007). Biomimetic Assembly of Zinc Oxide Nanorods onto Flexible Polymers. J. Am. Chem. Soc. 129, 45, 13776-13777, ISSN 0002-7863 (print), 1520-5126 (online)

Masuda, Y.; Kinoshita, N.; Sato, F.; Koumoto, K. (2006). Site-Selective Deposition and Morphology Control of UV- and Visible-Light-Emitting ZnO Crystals. Cryst. Growth Design 6, 75-78, ISSN 1528-7483.

Mikrajuddin, A.; Lenggoro, I. W.; Okuyama, K.; Shi, F. G. (2002). Luminescent Polymer Electrolytes. Prepared by Growing $\mathrm{ZnO}$ Nanoparticles in the Matrix of Polyethylene Glycol. J. Electrochem. Soc. 149, H107-H112, ISSN 0013-4651 (print), 1945-7111 (online).

Masuda, Y.; Sugiyama, T.; Seo, W. S.; Koumoto, K. (2003). Deposition Mechanism of Anatase $\mathrm{TiO}_{2}$ on Self-Assembled Monolayers from an Aqueous Solution. Chem. Mater. 15, 2469-2476, ISSN 0897-4756.

Naik, R. R.; Stringer, S. J.; Agarwal, G.; Jones, S. E.; Stone, M. O. (2002). Biomimetic synthesis and patterning of silver nanoparticles. Nat. Mater. 1, 169-172, ISSN 1476-1122 (print), 1476-4660 (online)

Pizem, H.; Gershevitz, O.; Goffer, Y.; Frimer, A. A.; Sukenik, C. N.; Sampathkumaran, U.; Milhet, X.; McIlwain, A.; De Guire, M. R.; Meador, M. A. B.; Sutter, J. K. (2005). Titania Deposition on PMR-15. Chem. Mater. 17, 3205-3213, ISSN 0897-4756.

Reuss, R. H.; Hopper, D. G.; Park, J. G. (2006). Macroelectronics. MRS Bull. 31, 447-450, ISSN 0883-7694.

Ravindran, S.; Ozkan, C. S. (2005). Self-assembly of $\mathrm{ZnO}$ nanoparticles to electrostatic coordination sites of functionalized carbon nanotubes. Nanotechnology 16, 11301136, ISSN 0957-4484 (print), 1361-6528 (online) 
Sarikaya. M.; Candan, T.; Alexk, Y. (2003). Molecular biomimetics: nanotechnology through biology. Nat. Mater. 9, 577-585, ISSN 1476-1122 (print), 1476-4660 (online)

Saito, N.; Haneda, H.; Seo, W. S.; Koumoto, K. (2001). Selective deposition of $\mathrm{ZnF}(\mathrm{OH})$ on self-assembled monolayers in $\mathrm{Zn}-\mathrm{NH}_{4} \mathrm{~F}$ aqueous solutions for micropatterning of zinc oxide. Langmuir 17, 1461-1469, ISSN 0743-7463.

Saito, N.; Haneda, H.; Sekiguchi, T.; Ohashi, N.; Sakaguchi, I.; Koumoto, K. (2002). LowTemperature Fabrication of Light-Emitting Zinc Oxide Micropatterns Using SelfAssembled Monolayers. Adv. Mater. 14, 418-421, ISSN 1521-4095.

Strohm, H.; Löbmann, P. (2005). Liquid-Phase Deposition of TiO2 on Polystyrene Latex Particles Functionalized by the Adsorption of Polyelectrolytes. Chem. Mater. 17, 6772-6780, ISSN 0897-4756.

Schmeer, G.; Riembauer, S.; Barthel, J. (1990). The influence of hydrophobic solvation on the alkaline hydrolysis of ethyl esters of polar substituted 2-methylpropionic acids in water. J. Solution Chem. 19, 1175-1189, ISSN 0095-9782 (print), 1572-8927 (online)

Tugulu, S.; Harms, M.; Fricke, M.; Volkmer, D.; Klok, H. A. (2006). Polymer Brushes as Ionotropic Matrices for the Directed Fabrication of Microstructured Calcite Thin Films. Angew. Chem., Int. Ed. 45, 44, 7458-7461, ISSN 1433-7851 (print), 1521-3773 (online)

Tamerler, C.; Oren, E. E.; Duman, M.; Venkatasubramanian, E.; Sarikaya, M. (2006). Adsorption Kinetics of an Engineered Gold Binding Peptide by Surface Plasmon Resonance Spectroscopy and a Quartz Crystal Microbalance. Langmuir 22, 77127718, ISSN 0743-7463.

Vollath, D.; Szabó, D. V.; Schlabach, S. (2004). Oxide/Polymer Nanocomposites as New Luminescent Materials. J. Nanoparticle Res. 6, 181-191, ISSN 1572-896X.

Wang, Z.; Chen, J. C.; Yang, P.; Yang, W. T. (2007) Biomimetic Synthesis of Gold Nanoparticles and Their aggregates using a Polypeptide Sequence. Appl. Organometal. Chem. 21, 8, 645-651, ISSN 0268-2605.

Wilmarth, W. K.; Haim, A. (1962). in Peroxide Reaction Mechanism, (Ed: Edwards, J. O.), Wiley Interscience, New York, p. 175.

Wu, H. S.; Jone, H. C. (1997). Kinetic Study and Characterization of Poly(acrylic acid) and Metal Oxide. Ind. Eng. Chem. Res. 36, 2006-2011, ISSN 0888-5885 (print), 1520-5045 (online)

Wang, Y.; Lai, H. H.; Bachman, M.; Sims, C. E.; Li, G. P.; Allbritton, N. L. (2005). Covalent micropatterning of poly(dimethylsiloxane) by photografting through a mask. Anal. Chem. 77, 7539-7546, ISSN 0003-2700.

Wu, X. L.; Siu, G. G.; Fu, C. L.; Ong, H. C. (2001). Photoluminescence and cathodoluminescence studies of stoichiometric and oxygen-deficient $\mathrm{ZnO}$ films. Appl. Phys. Lett. 78, 2285-2287, ISSN 0003-6951.

Xu, A. W.; Ma, Y.; Cölfen, H. (2007). Biomimetic Mineralization. J. Mater. Chem. 17, 5, 415449, ISSN 0959-9428 (print), 1364-5501 (online)

Yang, P.; Deng, J. Y.; Yang, W. T. (2003). Confined photo-catalytic oxidation: a fast surface hydrophilic modification method for polymeric materials. Polymer 44, 23, 71577164, ISSN 0032-3861. 
Yang, P.; Xie, J.; Yang, W. T. (2006). A Simple Method to Fabricate a Conductive Polymer Micropattern on an Organic Polymer Substrate. Macro. Rapid. Commn. 27, 418-423, ISSN 1521-3927.

Yang, P.; Yang, M.; Zou, S. L.; Xie, J. Y.; Yang, W. T. (2007). Positive and Negative TiO2 Micropatterns on Organic Polymer Substrates. J. Am. Chem. Soc. 129, 6, 1541-1552, ISSN 0002-7863 (print), 1520-5126 (online)

Yang, P.; Zhang, X.; Yang, B.; Zhao, H.; Chen, J.; Yang, W. T. (2005). Facile Preparation of a Patterned, Aminated Polymer Surface by UV-Light-Induced Surface Aminolysis. Adv. Funct. Mater. 15, 1415-1425, ISSN 1616-301X.

Yang, P.; Zhang, X.; Xie, J.; Chen, J.; Yang, W. T. (2006). Micro/Nanoscale Well and Channel Fabrication on Organic Polymer Substrates via a Combination of Photochemical and Alkaline Hydrolysis Etchings. Biomacromolecules 2006, 7, 10, 2770-2775, ISSN $1525-7797$.

Yang, P.; Zou, S. L.; Yang, W. T. (2008). Positive and Negative ZnO Micropatterning on Functionatized Polymer Surfaces. Small 4, 9, 1527-1536, ISSN 1613-6810 (print), 1613-6829 (online)

Zou, S; Bai, HD; Yang, P; Yang, WT. (2009) A Biomimetic Chemical Approach to Facile Preparation of Large-Area, Patterned, ZnO Quantum Dot/Polymer Nanocomposites on Flexible Plastics. Macro. Chem. Phys. 210, 18, 1519-1527, ISSN 1022-1352 (print), 1521-3935 (online)

Zhang, X.; Chen, J. C.; Yang, P.; Yang, W. T. (2005). Biomimetic Synthesis Silver Crystallite by Peptide AYSSGAPPMPPF Immobilized on PET film in vitro. J. Inorg. Biochem. 99, 8, 1692-1697, ISSN 0162-0134

Zhang, X.; Chen, J. C.; Yang, P.; Yang, W. T. (2006). Silver Biomimetic Mineralization in vitro with Inorganic-binding peptide AG4 (NPSSLFRYLPSD) Immobilized on PET Film. Chem. J. Chinese Universities 27, 1, 5-8, ISSN 0251-0790

Zubkov, T.; Lucassen, A. B.; Freeman, D.; Feldman, Y.; Cohen, S. R.; Evmenenko, G.; Dutta, P.; van der Boom, M. E. (2005). Photoinduced deprotection and $\mathrm{ZnO}$ patterning of hydroxyl-terminated siloxane-based monolayers. J. Phys. Chem. B 109, 14144-14153, ISSN 1089-5647.

Zhang, X.; Yang, P.; Yang, W. T.; Chen, J. C. (2008).The Bio-inspired Approach to Controllable Biomimetic Synthesis of Silver Nanoparticles in Organic Matrix of Chitosan and Silver-binding Peptide (NPSSLFRYLPSD). Mater. Sci. Eng. CBiomimetic Supramol. Syst. 28, 2, 237-242, ISSN 0928-4931 


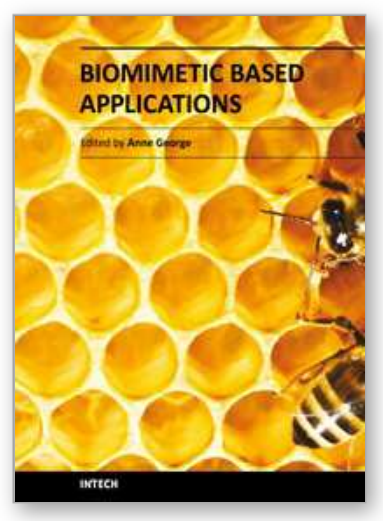

\author{
Biomimetic Based Applications \\ Edited by Prof. Marko Cavrak
}

ISBN 978-953-307-195-4

Hard cover, 572 pages

Publisher InTech

Published online 26, April, 2011

Published in print edition April, 2011

The interaction between cells, tissues and biomaterial surfaces are the highlights of the book "Biomimetic Based Applications". In this regard the effect of nanostructures and nanotopographies and their effect on the development of a new generation of biomaterials including advanced multifunctional scaffolds for tissue engineering are discussed. The 2 volumes contain articles that cover a wide spectrum of subject matter such as different aspects of the development of scaffolds and coatings with enhanced performance and bioactivity, including investigations of material surface-cell interactions.

\title{
How to reference
}

In order to correctly reference this scholarly work, feel free to copy and paste the following:

Peng Yang, Wantai Yang, Xu Zhang and Jinchun Chen (2011). Non-Calcium Inorganic Materials Fabrication by Surface-Immobilized Organic Molecular Template, Biomimetic Based Applications, Prof. Marko Cavrak (Ed.), ISBN: 978-953-307-195-4, InTech, Available from: http://www.intechopen.com/books/biomimetic-basedapplications/non-calcium-inorganic-materials-fabrication-by-surface-immobilized-organic-molecular-template

\section{INTECH}

open science | open minds

\section{InTech Europe}

University Campus STeP Ri

Slavka Krautzeka 83/A

51000 Rijeka, Croatia

Phone: +385 (51) 770447

Fax: +385 (51) 686166

www.intechopen.com

\section{InTech China}

Unit 405, Office Block, Hotel Equatorial Shanghai

No.65, Yan An Road (West), Shanghai, 200040, China

中国上海市延安西路65号上海国际贵都大饭店办公楼 405 单元

Phone: +86-21-62489820

Fax: +86-21-62489821 
(C) 2011 The Author(s). Licensee IntechOpen. This chapter is distributed under the terms of the Creative Commons Attribution-NonCommercialShareAlike-3.0 License, which permits use, distribution and reproduction for non-commercial purposes, provided the original is properly cited and derivative works building on this content are distributed under the same license. 